J. Korean Math. Soc. 48 (2011), No. 5, pp. 1017-1041

http://dx.doi.org/10.4134/JKMS.2011.48.5.1017

\title{
REPRESENTING SEQUENCES ON PARABOLIC BERGMAN SPACES
}

\author{
Yôsuke HishiKawA
}

\begin{abstract}
The parabolic Bergman space is the set of $L^{p}(\lambda)$-solutions of the parabolic operator $L^{(\alpha)}$. In this paper, we study representing sequences on parabolic Bergman spaces. We establish a discrete version of the reproducing formula on parabolic Bergman spaces by using fractional derivatives of the fundamental solution of the parabolic operator.
\end{abstract}

\section{Introduction}

Let $H$ be the upper half-space of the $(n+1)$-dimensional Euclidean space $\mathbb{R}^{n+1}(n \geq 1)$, that is, $H=\left\{(x, t) \in \mathbb{R}^{n+1} ; x \in \mathbb{R}^{n}, t>0\right\}$. For $0<\alpha \leq 1$, the parabolic operator $L^{(\alpha)}$ is defined by

$$
L^{(\alpha)}=\partial_{t}+\left(-\Delta_{x}\right)^{\alpha},
$$

where $\partial_{t}=\partial / \partial t$ and $\Delta_{x}$ is the Laplacian with respect to $x$. Let $C(H)$ be the set of all real-valued continuous functions on $H$. For a positive integer $k$, $C^{k}(H)$ denotes the set of all $k$ times continuously differentiable functions on $H$, and put $C^{\infty}(H)=\cap_{k} C^{k}(H)$. Furthermore, let $C_{c}^{\infty}(H)$ be the set of all functions in $C^{\infty}(H)$ with compact support. A function $u \in C(H)$ is said to be $L^{(\alpha)}$-harmonic if $L^{(\alpha)} u=0$ in the sense of distributions (for details, see Section 2 ). For $1 \leq p<\infty$ and $\lambda>-1, L^{p}(\lambda)$ is the set of all Lebesgue measurable functions $f$ on $H$ which satisfy

$$
\|f\|_{L^{p}(\lambda)}:=\left(\int_{H}|f(x, t)|^{p} t^{\lambda} d V(x, t)\right)^{\frac{1}{p}}<\infty,
$$

where $d V$ is the Lebesgue volume measure on $H$. The parabolic Bergman space $\boldsymbol{b}_{\alpha}^{p}(\lambda)$ is the set of all $L^{(\alpha)}$-harmonic functions $u$ on $H$ which belong to $L^{p}(\lambda)$. We remark that $\boldsymbol{b}_{\alpha}^{p}(\lambda)$ is a Banach space with the norm $\|\cdot\|_{L^{p}(\lambda)}$, and $\boldsymbol{b}_{1 / 2}^{p}(\lambda)$ coincides with the harmonic Bergman space of Ramey and Yi [9]. Also, we note that $\boldsymbol{b}_{\alpha}^{p}(\lambda)=\{0\}$ when $\lambda \leq-1$ (see Proposition 4.3 of [4]).

Received June 3, 2010.

2010 Mathematics Subject Classification. Primary 35K05; Secondary 26A33, 26D15.

Key words and phrases. parabolic operator of fractional order, Bergman space, representing sequence. 
Our aim of this paper is the study of representing sequences on parabolic Bergman spaces. In [3], we established the reproducing formula on parabolic Bergman spaces by using fractional derivatives of the fundamental solution of the parabolic operator $L^{(\alpha)}$. The reproducing formula of [3] is given by the integral of parabolic Bergman functions (see Theorem A below). In this paper, we study a discrete version of the reproducing formula, which is given by representing sequences.

We give some notations. For a real number $\kappa$, let $\mathcal{D}_{t}^{\kappa}=\left(-\partial_{t}\right)^{\kappa}$ be the fractional differential operator, and $W^{(\alpha)}$ the fundamental solution of the parabolic operator $L^{(\alpha)}$ (for the explicit definitions of $\mathcal{D}_{t}^{\kappa}$ and $W^{(\alpha)}$, see Section 2). In [3], the following reproducing formula on $\boldsymbol{b}_{\alpha}^{p}(\lambda)$ is given.

Theorem A (Theorem 4.7 of [3]). Let $0<\alpha \leq 1,1 \leq p<\infty$, and $\lambda>-1$. And let $\kappa>\frac{\lambda+1}{p}$ be a real number. Then the reproducing formula

$$
u(x, t)=C_{\kappa} \int_{H} u(x, t) \mathcal{D}_{t}^{\kappa} W^{(\alpha)}(x-y, t+s) t^{\kappa-1} d V(y, s)
$$

holds for all $u \in \boldsymbol{b}_{\alpha}^{p}(\lambda)$ and $(x, t) \in H$, where $C_{\kappa}=2^{\kappa} / \Gamma(\kappa)$ and $\Gamma(\cdot)$ is the gamma function. Moreover, the reproducing formula (1.1) also holds when $p=1$ and $\kappa=\lambda+1$.

To state our main results, we give some definitions. Let $0<\alpha \leq 1,1 \leq$ $p<\infty, \lambda>-1$, and $\kappa$ be a real number. Furthermore, let $\mathbb{X}=\left\{\left(x_{j}, t_{j}\right)\right\}$ be a sequence in $H$. For a sequence of real numbers $\left\{\eta_{j}\right\}$, we define a representing operator $U_{p, \mathbb{X}}^{\kappa}$ by

$$
U_{p, \mathbb{X}}^{\kappa}\left(\left\{\eta_{j}\right\}\right)(x, t)=\sum_{j} \eta_{j} t_{j}^{\frac{n}{2 \alpha}+\kappa-\left(\frac{n}{2 \alpha}+\lambda+1\right) \frac{1}{p}} \mathcal{D}_{t}^{\kappa} W^{(\alpha)}\left(x-x_{j}, t+t_{j}\right), \quad(x, t) \in H .
$$

By using the representing operator $U_{p, \mathbb{X}}^{\kappa}$, we define a representing sequence on $\boldsymbol{b}_{\alpha}^{p}(\lambda)$.

Definition 1. Let $0<\alpha \leq 1,1 \leq p<\infty, \lambda>-1$, and $\kappa$ be a real number. A sequence $\mathbb{X}=\left\{\left(x_{j}, t_{j}\right)\right\}$ in $H$ is called the $\boldsymbol{b}_{\alpha}^{p}(\lambda)$-representing sequence of order $\kappa$ if $U_{p, \mathbb{X}}^{\kappa}: \ell^{p} \rightarrow \boldsymbol{b}_{\alpha}^{p}(\lambda)$ is bounded and onto. Explicitly, a sequence $\mathbb{X}$ is called the $\boldsymbol{b}_{\alpha}^{p}(\lambda)$-representing sequence of order $\kappa$ if the following conditions are satisfied.

(1) For $\left\{\eta_{j}\right\} \in \ell^{p}$, the function $U_{p, \mathbb{X}}^{\kappa}\left(\left\{\eta_{j}\right\}\right)$ belongs to $\boldsymbol{b}_{\alpha}^{p}(\lambda)$, and there exists a constant $C>0$ such that $\left\|U_{p, \mathbb{X}}^{\kappa}\left(\left\{\eta_{j}\right\}\right)\right\|_{L^{p}(\lambda)} \leq C\left\|\left\{\eta_{j}\right\}\right\|_{\ell^{p}}$ for all $\left\{\eta_{j}\right\} \in \ell^{p}$.

(2) For $u \in \boldsymbol{b}_{\alpha}^{p}(\lambda)$, there exists $\left\{\eta_{j}\right\} \in \ell^{p}$ such that $u=U_{p, \mathbb{X}}^{\kappa}\left(\left\{\eta_{j}\right\}\right)$ on $H$. For $0<\delta<1$ and $(x, t) \in H$, an $\alpha$-parabolic cylinder $S_{\delta}^{(\alpha)}$ is defined by

$$
S_{\delta}^{(\alpha)}(x, t)=\left\{(y, s) \in H ;|y-x|<\left(\frac{2 \delta}{1-\delta^{2}} t\right)^{1 / 2 \alpha}, \frac{1-\delta}{1+\delta} t<s<\frac{1+\delta}{1-\delta} t\right\} .
$$


A sequence $\left\{\left(x_{j}, t_{j}\right)\right\}$ in $H$ is said to be $\delta$-separated in the $\alpha$-parabolic sense if $S_{\delta}^{(\alpha)}\left(x_{j}, t_{j}\right) \cap S_{\delta}^{(\alpha)}\left(x_{i}, t_{i}\right)=\emptyset$ for $j \neq i$. Furthermore, a sequence $\left\{\left(x_{j}, t_{j}\right)\right\}$ in $H$ is said to be a $\delta$-lattice in the $\alpha$-parabolic sense if $\left\{\left(x_{j}, t_{j}\right)\right\}$ satisfies the following;

(i) $\cup_{j} S_{\delta}^{(\alpha)}\left(x_{j}, t_{j}\right)=H$.

(ii) For some $0<\varepsilon<\delta,\left\{\left(x_{j}, t_{j}\right)\right\}$ is $\varepsilon$-separated in the $\alpha$-parabolic sense.

The following theorems are the main results of this paper.

Theorem 1. Let $0<\alpha \leq 1,1<p<\infty, \lambda>-1$, and $\kappa>\frac{\lambda+1}{p}$ be a real number. Furthermore, let $\mathbb{X}=\left\{\left(x_{j}, t_{j}\right)\right\}$ be a sequence in $H$. Then, $U_{p, \mathbb{X}}^{\kappa}$ satisfies the condition (1) of Definition 1 if and only if for any $0<\delta<1$, there exists $K \in \mathbb{N}$ such that $\mathbb{X}=\mathbb{X}_{1} \cup \cdots \cup \mathbb{X}_{K}$ and each sequence $\mathbb{X}_{i}$ is $\delta$-separated in the $\alpha$-parabolic sense. The "if" part also holds when $p=1$.

Theorem 2. Let $0<\alpha \leq 1,1 \leq p<\infty, \lambda>-1$, and $\kappa>\frac{\lambda+1}{p}$ be a real number. Then, there exists $0<\delta_{0}<1$ such that if a sequence $\mathbb{X}$ in $H$ is a $\delta$ lattice in the $\alpha$-parabolic sense with $0<\delta \leq \delta_{0}$, then $\mathbb{X}$ is a $\boldsymbol{b}_{\alpha}^{p}(\lambda)$-representing sequence of order $\kappa$.

By Theorem 2 and the open mapping theorem, we obtain the following corollary.

Corollary 1. Let $0<\alpha \leq 1,1 \leq p<\infty, \lambda>-1$, and $\kappa>\frac{\lambda+1}{p}$ be $a$ real number. Then, there exists a sequence $\mathbb{X}=\left\{\left(x_{j}, t_{j}\right)\right\}$ in $H$ such that the following properties hold:

(1) For $\left\{\eta_{j}\right\} \in \ell^{p}$, the function $u=U_{p, \mathbb{X}}^{\kappa}\left(\left\{\eta_{j}\right\}\right)$ belongs to $\boldsymbol{b}_{\alpha}^{p}(\lambda)$, and there exists a constant $C>0$ such that

for all $\left\{\eta_{j}\right\} \in \ell^{p}$.

$$
\|u\|_{L^{p}(\lambda)} \leq C\left\|\left\{\eta_{j}\right\}\right\|_{\ell^{p}}
$$

(2) For $u \in \boldsymbol{b}_{\alpha}^{p}(\lambda)$, there exists $\left\{\eta_{j}\right\} \in \ell^{p}$ such that $u=U_{p, \mathbb{X}}^{\kappa}\left(\left\{\eta_{j}\right\}\right)$ on $H$. Moreover, there exists a constant $C>0$ independent of $u$ such that

$$
\left\|\left\{\eta_{j}\right\}\right\|_{\ell^{p}} \leq C\|u\|_{L^{p}(\lambda)} .
$$

This paper is constructed as follows. In Section 2, we present basic properties of parabolic Bergman functions. In Section 3, we study Carleson inequalities on parabolic Bergman spaces with fractional derivatives. The results of Carleson inequalities shall be used for the proof of Theorem 1. In Section 4, we give the proof of Theorem 1. In Section 5, we give our representation theorem, that is, we show Theorem 2. In Section 6, we give the definition of $\boldsymbol{b}_{\alpha}^{p}(\lambda)$-sampling sequences, and we show that $\boldsymbol{b}_{\alpha}^{p}(\lambda)$-representing sequences are closely related to $\boldsymbol{b}_{\alpha}^{p}(\lambda)$-sampling sequences.

Throughout this paper, we will denote by $C$ a positive constant whose value may not necessarily be the same at each occurrence. 


\section{Preliminaries}

In this section, we present preliminary results. We recall the definition of $L^{(\alpha)}$-harmonic functions. We describe the operator $\left(-\Delta_{x}\right)^{\alpha}$. Since the case $\alpha=1$ is trivial, we only describe the case $0<\alpha<1$. For $0<\alpha<1,\left(-\Delta_{x}\right)^{\alpha}$ is the convolution operator defined by

$$
\left(-\Delta_{x}\right)^{\alpha} \psi(x, t)=-c_{n, \alpha} \lim _{\delta \rightarrow 0^{+}} \int_{|y-x|>\delta}(\psi(y, t)-\psi(x, t))|y-x|^{-n-2 \alpha} d y
$$

for all $\psi \in C_{c}^{\infty}(H)$ and $(x, t) \in H$, where $c_{n, \alpha}=-4^{\alpha} \pi^{-n / 2} \Gamma((n+2 \alpha) / 2) / \Gamma(-\alpha)$ $>0$. A function $u \in C(H)$ is said to be $L^{(\alpha)}$-harmonic on $H$ if $u$ satisfies the following condition: for every $\psi \in C_{c}^{\infty}(H)$,

$$
\int_{H}\left|u \cdot \widetilde{L}^{(\alpha)} \psi\right| d V<\infty \text { and } \int_{H} u \cdot \widetilde{L}^{(\alpha)} \psi d V=0
$$

where $\widetilde{L}^{(\alpha)}=-\partial_{t}+\left(-\Delta_{x}\right)^{\alpha}$ is the adjoint operator of $L^{(\alpha)}$. By $(2.1)$ and the compactness of $\operatorname{supp}(\psi)$ (the support of $\psi$ ), there exist $0<t_{1}<t_{2}<$ $\infty$ and a constant $C>0$ such that $\operatorname{supp}\left(\widetilde{L}^{(\alpha)} \psi\right) \subset S=\mathbb{R}^{n} \times\left[t_{1}, t_{2}\right]$ and $\left|\widetilde{L}^{(\alpha)} \psi(x, t)\right| \leq C(1+|x|)^{-n-2 \alpha}$ for all $(x, t) \in S$. Thus, the integrability condition $\int_{H}\left|u \cdot \widetilde{L}^{(\alpha)} \psi\right| d V<\infty$ is equivalent to the following: for any $0<t_{1}<$ $t_{2}<\infty$

$$
\int_{t_{1}}^{t_{2}} \int_{\mathbb{R}^{n}}|u(x, t)|(1+|x|)^{-n-2 \alpha} d V(x, t)<\infty .
$$

We introduce the fundamental solution of $L^{(\alpha)}$. For $x \in \mathbb{R}^{n}$, the fundamental solution $W^{(\alpha)}$ of $L^{(\alpha)}$ is defined by

$$
W^{(\alpha)}(x, t)= \begin{cases}\frac{1}{(2 \pi)^{n}} \int_{\mathbb{R}^{n}} \exp \left(-t|\xi|^{2 \alpha}+\sqrt{-1} x \cdot \xi\right) d \xi, & t>0 \\ 0, & t \leq 0\end{cases}
$$

where $x \cdot \xi$ denotes the inner product on $\mathbb{R}^{n}$. It is known that $W^{(\alpha)}$ is $L^{(\alpha)}$ harmonic on $H$ and $W^{(\alpha)} \in C^{\infty}(H)$.

We present definitions of fractional integral and differential operators. Let $C\left(\mathbb{R}_{+}\right)$be the set of all continuous functions on $\mathbb{R}_{+}=(0, \infty)$. For a positive real number $\kappa$, let $\mathcal{F} \mathcal{C}^{-\kappa}$ be the set of all functions $\varphi \in C\left(\mathbb{R}_{+}\right)$such that there exists a constant $\varepsilon>0$ with $\varphi(t)=O\left(t^{-\kappa-\varepsilon}\right)$ as $t \rightarrow \infty$. We remark that $\mathcal{F C}^{-\nu} \subset \mathcal{F C}^{-\kappa}$ if $0<\kappa \leq \nu$. For $\varphi \in \mathcal{F C}^{-\kappa}$, we can define the fractional integral of $\varphi$ with order $\kappa$ by

$$
\mathcal{D}_{t}^{-\kappa} \varphi(t)=\frac{1}{\Gamma(\kappa)} \int_{0}^{\infty} \tau^{\kappa-1} \varphi(t+\tau) d \tau, \quad t \in \mathbb{R}_{+} .
$$

Furthermore, let $\mathcal{F C} \mathcal{C}^{\kappa}$ be the set of all functions $\varphi \in C\left(\mathbb{R}_{+}\right)$such that $\partial_{t}^{\lceil\kappa\rceil} \varphi \in$ $\mathcal{F C}^{-(\lceil\kappa\rceil-\kappa)}$, where $\lceil\kappa\rceil$ is the smallest integer greater than or equal to $\kappa$. In 
particular, we will write $\mathcal{F} \mathcal{C}^{0}=C\left(\mathbb{R}_{+}\right)$. For $\varphi \in \mathcal{F} \mathcal{C}^{\kappa}$, we can also define the fractional derivative of $\varphi$ with order $\kappa$ by

$$
\mathcal{D}_{t}^{\kappa} \varphi(t)=\mathcal{D}_{t}^{-(\lceil\kappa\rceil-\kappa)}\left(-\partial_{t}\right)^{\lceil\kappa\rceil} \varphi(t), t \in \mathbb{R}_{+} .
$$

Also, we define $\mathcal{D}_{t}^{0} \varphi=\varphi$. We may often call both (2.3) and (2.4) the fractional derivative of $\varphi$ with order $\kappa$. Moreover, we call $\mathcal{D}_{t}^{\kappa}$ the fractional differential operator with order $\kappa$.

We describe basic properties of fractional derivatives of the fundamental solution $W^{(\alpha)}$, which are given in [3]. Let $\mathbb{N}_{0}=\mathbb{N} \cup\{0\}$. For a multi-index $\beta=\left(\beta_{1}, \ldots, \beta_{n}\right) \in \mathbb{N}_{0}^{n}$, let $\partial_{x}^{\beta}=\partial^{|\beta|} / \partial x_{1}{ }^{\beta_{1}} \cdots \partial x_{n}{ }^{\beta_{n}}$, where $|\beta|=\beta_{1}+\cdots+\beta_{n}$. The following lemma is given by Theorem 3.1 of [3].

Lemma 2.1 (Theorem 3.1 of [3]). Let $0<\alpha \leq 1, \beta \in \mathbb{N}_{0}^{n}$, and $\kappa>-\frac{n}{2 \alpha}$ be a real number. Then, the following statements hold.

(1) The derivatives $\partial_{x}^{\beta} \mathcal{D}_{t}^{\kappa} W^{(\alpha)}$ and $\mathcal{D}_{t}^{\kappa} \partial_{x}^{\beta} W^{(\alpha)}$ are well-defined, and

$$
\partial_{x}^{\beta} \mathcal{D}_{t}^{\kappa} W^{(\alpha)}(x, t)=\mathcal{D}_{t}^{\kappa} \partial_{x}^{\beta} W^{(\alpha)}(x, t)
$$

for all $(x, t) \in H$. Moreover, there exists a constant $C>0$ such that

$$
\left|\partial_{x}^{\beta} \mathcal{D}_{t}^{\kappa} W^{(\alpha)}(x, t)\right| \leq C\left(t+|x|^{2 \alpha}\right)^{-\frac{n+|\beta|}{2 \alpha}-\kappa}
$$

for all $(x, t) \in H$.

(2) Let $\nu$ be a real number such that $\kappa+\nu>-\frac{n}{2 \alpha}$. Then,

$$
\mathcal{D}_{t}^{\nu} \partial_{x}^{\beta} \mathcal{D}_{t}^{\kappa} W^{(\alpha)}(x, t)=\partial_{x}^{\beta} \mathcal{D}_{t}^{\kappa+\nu} W^{(\alpha)}(x, t)
$$

for all $(x, t) \in H$.

(3) $\partial_{x}^{\beta} \mathcal{D}_{t}^{\kappa} W^{(\alpha)}$ is $L^{(\alpha)}$-harmonic on $H$.

We also note that $\partial_{x}^{\beta} \mathcal{D}_{t}^{\kappa} W^{(\alpha)}(-x, t)=(-1)^{|\beta|} \partial_{x}^{\beta} \mathcal{D}_{t}^{\kappa} W^{(\alpha)}(x, t)$ by the definition of $W^{(\alpha)}$.

For $(x, t) \in H$, the $\alpha$-parabolic Carleson box $Q^{(\alpha)}$ is defined by

$$
Q^{(\alpha)}(x, t)=\left\{(y, s) \in H ;\left|y_{j}-x_{j}\right|<2^{-1} t^{1 / 2 \alpha}(1 \leq j \leq n), t \leq s \leq 2 t\right\} .
$$

Clearly, we have $V\left(Q^{(\alpha)}(x, t)\right)=C t^{\frac{n}{2 \alpha}+1}$ for all $(x, t) \in H$. The $\alpha$-parabolic Carleson boxes are used in the study of Carleson inequalities on $\boldsymbol{b}_{\alpha}^{p}(\lambda)$ in Section 3. The following lemma shows that $Q^{(\alpha)}$ is nearly $S_{\delta}^{(\alpha)}$.

Lemma 2.2 (Lemma 3.2 of [7]). For any $0<\delta<1$, there exist positive integers $M_{1}, M_{2}$ and constants $C_{1}, C_{2}>0$ with the following properties:

(1) For each $(y, s) \in H$, there exist points $\left\{\left(y_{j}, s_{j}\right)\right\} \subset H\left(j=1,2, \ldots, M_{1}\right)$ such that

$$
Q^{(\alpha)}(y, s) \subset \bigcup_{j=1}^{M_{1}} S_{\delta}^{(\alpha)}\left(y_{j}, s_{j}\right) \text { and } s \leq s_{j} \leq C_{1} s
$$


(2) For each $(y, s) \in H$, there exist points $\left\{\left(y_{j}, s_{j}\right)\right\} \subset H\left(j=1,2, \ldots, M_{2}\right)$ such that

$$
S_{\delta}^{(\alpha)}(y, s) \subset \bigcup_{j=1}^{M_{2}} Q^{(\alpha)}\left(y_{j}, s_{j}\right) \text { and } \frac{1}{C_{2}} s \leq s_{j} \leq C_{2} s .
$$

In [3], we also give the lower estimate of fractional derivatives of $W^{(\alpha)}$ on $Q^{(\alpha)}$.

Lemma 2.3 (Proposition 3.2 of [3]). Let $0<\alpha \leq 1, \beta \in \mathbb{N}_{0}^{n}$, and $\kappa>-\frac{n}{2 \alpha}$ be a real number. If each $\beta_{j}$ is even, then there exist constants $\rho, C>0$ such that

$$
C^{-1} s^{-\frac{n+|\beta|}{2 \alpha}-\kappa} \leq\left|\partial_{x}^{\beta} \mathcal{D}_{t}^{\kappa} W^{(\alpha)}(x-y, t+s)\right| \leq C s^{-\frac{n+|\beta|}{2 \alpha}-\kappa}
$$

for all $(x, t) \in Q^{(\alpha)}(y, \rho s)$, where $\rho$ and $C$ depend on $n, \alpha, \beta$ and $\kappa$.

We describe basic properties of fractional derivatives of parabolic Bergman functions.

Lemma 2.4 (Proposition 4.1 of [3]). Let $0<\alpha \leq 1,1 \leq p<\infty, \lambda>-1$, $\beta \in \mathbb{N}_{0}^{n}$, and $\kappa>-\left(\frac{n}{2 \alpha}+\lambda+1\right) \frac{1}{p}$ be a real number. If $u \in \boldsymbol{b}_{\alpha}^{p}(\lambda)$, then the following statements hold:

(1) The derivatives $\partial_{x}^{\beta} \mathcal{D}_{t}^{\kappa} u$ and $\mathcal{D}_{t}^{\kappa} \partial_{x}^{\beta} u$ are well-defined, and

$$
\partial_{x}^{\beta} \mathcal{D}_{t}^{\kappa} u(x, t)=\mathcal{D}_{t}^{\kappa} \partial_{x}^{\beta} u(x, t)
$$

for all $(x, t) \in H$. Moreover, there exists a constant $C>0$ such that

$$
\left|\partial_{x}^{\beta} \mathcal{D}_{t}^{\kappa} u(x, t)\right| \leq C t^{-\frac{|\beta|}{2 \alpha}-\kappa-\left(\frac{n}{2 \alpha}+\lambda+1\right) \frac{1}{p}}\|u\|_{L^{p}(\lambda)}
$$

for all $(x, t) \in H$.

(2) Let $\nu$ be a real number such that $\kappa+\nu>-\left(\frac{n}{2 \alpha}+\lambda+1\right) \frac{1}{p}$. Then,

$$
\mathcal{D}_{t}^{\nu} \partial_{x}^{\beta} \mathcal{D}_{t}^{\kappa} u(x, t)=\partial_{x}^{\beta} \mathcal{D}_{t}^{\kappa+\nu} u(x, t)
$$

for all $(x, t) \in H$.

(3) $\partial_{x}^{\beta} \mathcal{D}_{t}^{\kappa} u$ is $L^{(\alpha)}$-harmonic on $H$.

In [3], the following reproducing formula on parabolic Bergman spaces is given, which is the generalization of Theorem $\mathrm{A}$.

Lemma 2.5 (Theorem 5.2 of [3]). Let $0<\alpha \leq 1,1<p<\infty$, and $\lambda>-1$. Also, let $\nu>-\frac{\lambda+1}{p}$ and $\kappa>\frac{\lambda+1}{p}$ be real numbers. Then, the reproducing formula

$$
u(x, t)=C_{\nu+\kappa} \int_{H} \mathcal{D}_{t}^{\nu} u(y, s) \mathcal{D}_{t}^{\kappa} W^{(\alpha)}(x-y, t+s) s^{\nu+\kappa-1} d V(y, s)
$$

holds for all $u \in \boldsymbol{b}_{\alpha}^{p}(\lambda)$ and $(x, t) \in H$. Moreover, the reproducing formula (2.5) also holds when $p=1$ and $\kappa=\lambda+1$.

We need the following duality theorem on parabolic Bergman spaces, which is used in Section 4. 
Lemma 2.6 ((2) of Corollary 3.2 of [4]). Let $0<\alpha \leq 1,1<p<\infty$, and $\lambda>-1$. And let $q$ be the exponent conjugate to $p$. Then, $\left(\boldsymbol{b}_{\alpha}^{p}(\lambda)\right)^{*} \simeq \boldsymbol{b}_{\alpha}^{q}(\lambda)$ under the integral pairing

$$
\langle u, v\rangle_{\lambda}=\int_{H} u(x, t) v(x, t) t^{\lambda} d V(x, t), u \in \boldsymbol{b}_{\alpha}^{p}(\lambda), v \in \boldsymbol{b}_{\alpha}^{q}(\lambda) .
$$

For $\kappa, \theta, \sigma \in \mathbb{R}$ and $f \in L^{p}(\sigma)$, let

$$
\Psi_{\alpha}^{\kappa, \theta} f(x, t):=\int_{H}|f(y, s)| \frac{s^{\theta}}{\left(t+s+|x-y|^{2 \alpha}\right)^{\frac{n}{2 \alpha}+\kappa}} d V(y, s), \quad(x, t) \in H .
$$

In the proof of (1) of Theorem 3.1 of [4], the authors show the following result, which is used in our argument.

Lemma 2.7 (Theorem 3.1 of [4]). Let $0<\alpha \leq 1,1 \leq p<\infty$, and $\sigma \in \mathbb{R}$. Suppose that $\kappa, \theta \in \mathbb{R}$ with $\kappa>-\frac{n}{2 \alpha}$ satisfy

$$
\sigma-\theta p<p-1<\kappa p+\sigma-\theta p .
$$

Then, there exists a constant $C>0$ such that

$$
\left\|\Psi_{\alpha}^{\kappa, \theta} f\right\|_{L^{p}(\eta)} \leq C\|f\|_{L^{p}(\sigma)}
$$

for all $f \in L^{p}(\sigma)$, where $\eta=(\kappa-\theta-1) p+\sigma$.

\section{Carleson inequalities with fractional derivatives}

Let $\gamma \in \mathbb{N}_{0}^{n}$ and $\nu, \rho, \sigma \in \mathbb{R}$. We say that a positive Borel measure $\mu$ on $H$ satisfies a Carleson inequality on $\boldsymbol{b}_{\alpha}^{p}(\lambda)$ with fractional derivatives if there exists a constant $C>0$ such that

$$
\int_{H}\left|\partial_{x}^{\gamma} \mathcal{D}_{t}^{\nu} u(x, t)\right|^{p} d \mu(x, t) \leq C \int_{H}\left|\mathcal{D}_{t}^{\rho} u(x, t)\right|^{p} t^{\sigma} d V(x, t)
$$

for all $u \in \boldsymbol{b}_{\alpha}^{p}(\lambda)$. In this section, we study the Carleson inequalities on $\boldsymbol{b}_{\alpha}^{p}(\lambda)$ with fractional derivatives. A result obtained by the study of the Carleson inequalities on $\boldsymbol{b}_{\alpha}^{p}(\lambda)$ should be used for the proof of Theorem 1. First, we show the following lemma, which is an important tool for the proof of the main theorem in this section.

Lemma 3.1. Let $0<\alpha \leq 1$ and $\theta, c \in \mathbb{R}$. If there exist constants $\varepsilon, M>0$ such that

$$
\frac{n}{2 \alpha}<\theta+\varepsilon<c, \text { and } \mu\left(Q^{(\alpha)}(x, t)\right) \leq M t^{\varepsilon}
$$

for all $(x, t) \in H$, then there exists a constant $C>0$ such that

$$
\int_{H} \frac{s^{\theta}}{\left(t+s+|x-y|^{2 \alpha}\right)^{c}} d \mu(y, s) \leq C t^{\theta+\varepsilon-c}
$$

for all $(x, t) \in H$. 
Proof. Let $(x, t) \in H$ be fixed. For a multi-index $\gamma=\left(\gamma_{1}, \ldots, \gamma_{n}\right) \in \mathbb{Z}^{n}$ and $m \in \mathbb{Z}$, we define $Q_{\gamma, m}$ by

$$
\begin{aligned}
Q_{\gamma, m}:=\{ & (y, s) \in H ; \gamma_{j}\left(2^{m} t\right)^{\frac{1}{2 \alpha}} \leq x_{j}-y_{j}<\left(\gamma_{j}+1\right)\left(2^{m} t\right)^{\frac{1}{2 \alpha}}(1 \leq j \leq n), \\
& \left.2^{m} t \leq s<2^{m+1} t\right\} .
\end{aligned}
$$

Then, $\left\{Q_{\gamma, m}\right\}$ is a set of $\alpha$-parabolic Carleson boxes, and $H=\cup Q_{\gamma, m}$. Moreover, since $\mu\left(Q^{(\alpha)}(x, t)\right) \leq M t^{\varepsilon}$, we have $\mu\left(Q_{\gamma, m}\right) \leq M\left(2^{m} t\right)^{\varepsilon}$. Hence, we obtain

$$
\begin{aligned}
& \int_{H} \frac{s^{\theta}}{\left(t+s+|x-y|^{2 \alpha}\right)^{c}} d \mu(y, s) \\
= & \int_{\cup Q_{\gamma, m}} \frac{s^{\theta}}{\left(t+s+|x-y|^{2 \alpha}\right)^{c}} d \mu(y, s) \\
= & \sum_{m \in \mathbb{Z}} \sum_{\gamma \in \mathbb{Z}^{n}} \int_{Q_{\gamma, m}} \frac{s^{\theta}}{\left(t+s+|x-y|^{2 \alpha}\right)^{c}} d \mu(y, s) \\
\leq & C \sum_{m \in \mathbb{Z}} \sum_{\gamma \in \mathbb{Z}^{n}} \frac{\left(2^{m} t\right)^{\theta}}{\left(t+2^{m} t+2^{m} t\left(\gamma_{1}^{2}+\cdots+\gamma_{n}^{2}\right)^{\alpha}\right)^{c}} \mu\left(Q_{\gamma, m}\right) \\
\leq & C M \sum_{m \in \mathbb{Z}} \sum_{\gamma \in \mathbb{Z}^{n}} \frac{\left(2^{m} t\right)^{\theta+\varepsilon}}{\left(t+2^{m} t+2^{m} t\left(\gamma_{1}^{2}+\cdots+\gamma_{n}^{2}\right)^{\alpha}\right)^{c}} \\
= & C M t^{\theta+\varepsilon-c} \sum_{m \in \mathbb{Z}} 2^{m(\theta+\varepsilon-c)} \sum_{\gamma \in \mathbb{Z}^{n}} \frac{1}{\left(2^{-m}+1+\left(\gamma_{1}^{2}+\cdots+\gamma_{n}^{2}\right)^{\alpha}\right)^{c}} .
\end{aligned}
$$

By elementary calculations, we have

$$
\begin{aligned}
& \sum_{\gamma \in \mathbb{Z}^{n}} \frac{1}{\left(2^{-m}+1+\left(\gamma_{1}^{2}+\cdots+\gamma_{n}^{2}\right)^{\alpha}\right)^{c}} \\
\leq & C \int_{\mathbb{R}^{n}} \frac{1}{\left(2^{-m}+1+|x|^{2 \alpha}\right)^{c}} d x \\
\leq & C\left(2^{-m}+1\right)^{\frac{n}{2 \alpha}-c} \int_{0}^{\infty} \frac{\eta^{\frac{n}{2 \alpha c}}-1}{1+\eta} d \eta \leq C\left(2^{-m}+1\right)^{\frac{n}{2 \alpha}-c} .
\end{aligned}
$$

Since $2^{-m}+1 \geq 1(m \geq 0)$ and $2^{-m}+1 \geq 2^{-m}(m<0)$, we obtain

$$
\begin{aligned}
& \int_{H} \frac{s^{\theta}}{\left(t+s+|x-y|^{2 \alpha}\right)^{c}} d \mu(y, s) \\
\leq & C t^{\theta+\varepsilon-c}\left\{\sum_{m \geq 0} 2^{m(\theta+\varepsilon-c)}+\sum_{m<0} 2^{m\left(\theta+\varepsilon-\frac{n}{2 \alpha}\right)}\right\} .
\end{aligned}
$$


Because $\frac{n}{2 \alpha}<\theta+\varepsilon<c$, we conclude

$$
\int_{H} \frac{s^{\theta}}{\left(t+s+|x-y|^{2 \alpha}\right)^{c}} d \mu(y, s) \leq C t^{\theta+\varepsilon-c} .
$$

Thus, this completes the proof.

If $\mu$ is the Lebesgue volume measure $V$ on $H$, then there exists a constant $M>0$ such that $\mu\left(Q^{(\alpha)}(x, t)\right)=M t^{\frac{n}{2 \alpha}+1}$ for all $(x, t) \in H$. Therefore by Lemma 3.1, we have the following corollary.

Corollary 3.2. Let $0<\alpha \leq 1$ and $\theta>-1$. If a constant $c>0$ satisfies the condition $\frac{n}{2 \alpha}+\theta+1<c$, then there exists a constant $C>0$ such that

$$
\int_{H} \frac{s^{\theta}}{\left(t+s+|x-y|^{2 \alpha}\right)^{c}} d V(y, s) \leq C t^{\frac{n}{2 \alpha}+\theta+1-c}
$$

for all $(x, t) \in H$.

In order to prove the main theorem of this section, we also give the following lemma.

Lemma 3.3. Let $0<\alpha \leq 1,1 \leq p<\infty$, and $\lambda>-1$. Furthermore, let $\gamma \in \mathbb{N}_{0}^{n}, \nu, \rho>-\frac{\lambda+1}{p}, \sigma \in \mathbb{R}$, and $\mu$ be a positive Borel measure on $H$. Suppose $\eta \in \mathbb{R}$ and $\kappa>\frac{\lambda+1}{p}$ satisfy the condition

$$
-\frac{|\gamma|}{2 \alpha}-\nu<\frac{\eta}{p}-\rho<\kappa .
$$

If there exists a constant $M>0$ such that

$$
\begin{aligned}
& F_{p}(x, t) \\
:= & s^{\rho+\kappa-\sigma-1+\frac{\eta(p-1)}{p}} \int_{H}\left|\partial_{x}^{\gamma} \mathcal{D}_{t}^{\nu+\kappa} W^{(\alpha)}(x-y, t+s)\right| t^{-\left(\frac{|\gamma|}{2 \alpha}+\nu-\rho+\frac{\eta}{p}\right)(p-1)} d \mu(x, t) \\
\leq & M
\end{aligned}
$$

for all $(y, s) \in H$, then there exists a constant $C>0$ such that

$$
\int_{H}\left|\partial_{x}^{\gamma} \mathcal{D}_{t}^{\nu} u(x, t)\right|^{p} d \mu(x, t) \leq C \int_{H}\left|\mathcal{D}_{t}^{\rho} u(x, t)\right|^{p} t^{\sigma} d V(x, t)
$$

for all $u \in \boldsymbol{b}_{\alpha}^{p}(\lambda)$.

Proof. Let $u \in \boldsymbol{b}_{\alpha}^{p}(\lambda)$. Differentiating through the integral (2.5), Lemma 2.5 and the Fubini theorem imply that

$$
\begin{aligned}
& \partial_{x}^{\gamma} \mathcal{D}_{t}^{\nu} u(x, t) \\
= & C_{\rho+\kappa} \int_{H} \mathcal{D}_{t}^{\rho} u(y, s) \partial_{x}^{\gamma} \mathcal{D}_{t}^{\nu+\kappa} W^{(\alpha)}(x-y, t+s) s^{\rho+\kappa-1} d V(y, s), \quad(x, t) \in H .
\end{aligned}
$$


We show (3.1) when $1<p<\infty$. Let $q$ be the exponent conjugate to $p$. Then by (3) and the Hölder inequality, we have

$$
\begin{aligned}
& \left|\partial_{x}^{\gamma} \mathcal{D}_{t}^{\nu} u(x, t)\right|^{p} \\
\leq & C\left(\int_{H}\left|\mathcal{D}_{t}^{\rho} u(y, s)\right|\left|\partial_{x}^{\gamma} \mathcal{D}_{t}^{\nu+\kappa} W^{(\alpha)}(x-y, t+s)\right| s^{\rho+\kappa-1} d V(y, s)\right)^{p} \\
= & C\left(\int_{H}\left|\mathcal{D}_{t}^{\rho} u(y, s)\right|\left|\partial_{x}^{\gamma} \mathcal{D}_{t}^{\nu+\kappa} W^{(\alpha)}(x-y, t+s)\right| s^{\frac{\eta}{p q}} s^{-\frac{\eta}{p q}} s^{\rho+\kappa-1} d V(y, s)\right)^{p} \\
\leq & C \int_{H}\left|\mathcal{D}_{t}^{\rho} u(y, s)\right|^{p}\left|\partial_{x}^{\gamma} \mathcal{D}_{t}^{\nu+\kappa} W^{(\alpha)}(x-y, t+s)\right| s^{\rho+\kappa-1+\frac{\eta}{q}} d V(y, s) \\
& \times\left(\int_{H}\left|\partial_{x}^{\gamma} \mathcal{D}_{t}^{\nu+\kappa} W^{(\alpha)}(x-y, t+s)\right| s^{\rho+\kappa-1-\frac{\eta}{p}} d V(y, s)\right)^{\frac{p}{q}} .
\end{aligned}
$$

Since $-\frac{|\gamma|}{2 \alpha}-\nu<\frac{\eta}{p}-\rho<\kappa$, Corollary 3.2 implies that

$$
\left(\int_{H}\left|\partial_{x}^{\gamma} \mathcal{D}_{t}^{\nu+\kappa} W^{(\alpha)}(x-y, t+s)\right| s^{\rho+\kappa-1-\frac{\eta}{p}} d V(y, s)\right)^{\frac{p}{q}} \leq C t^{-\left(\frac{|\gamma|}{2 \alpha}+\nu-\rho+\frac{\eta}{p}\right) \frac{p}{q}}
$$

for all $(x, t) \in H$. Hence by the Fubini theorem, we obtain

$$
\int_{H}\left|\partial_{x}^{\gamma} \mathcal{D}_{t}^{\nu} u(x, t)\right|^{p} d \mu(x, t) \leq C \int_{H}\left|\mathcal{D}_{t}^{\rho} u(y, s)\right|^{p} F_{p}(y, s) s^{\sigma} d V(y, s) .
$$

Because $F_{p}(y, s) \leq M$ for all $(y, s) \in H$, we conclude (3.1) when $1<p<\infty$.

We show (3.1) when $p=1$. By (3) and the Fubini theorem, we have

$$
\int_{H}\left|\partial_{x}^{\gamma} \mathcal{D}_{t}^{\nu} u(x, t)\right| d \mu(x, t) \leq C \int_{H}\left|\mathcal{D}_{t}^{\rho} u(y, s)\right| F_{1}(y, s) s^{\sigma} d V(y, s) .
$$

Because $F_{1}(y, s) \leq M$ for all $(y, s) \in H$, we conclude (3.1) when $p=1$.

Now, we show the main theorem of this section. We give necessary and sufficient conditions for a measure $\mu$ to satisfy the Carleson inequality on $\boldsymbol{b}_{\alpha}^{p}(\lambda)$ with fractional derivatives.

Theorem 3.4. Let $0<\alpha \leq 1,1 \leq p<\infty, \lambda>-1$, and $\mu$ be a positive Borel measure on $H$. Furthermore, let $\gamma \in \mathbb{N}_{0}^{n}, \nu, \rho>-\frac{\lambda+1}{p}$, and $\sigma>-1$ be real numbers such that $\sigma+1+\left(\frac{|\gamma|}{2 \alpha}+\nu-\rho\right) p>0$. Then, the following statements are equivalent:

(1) There exists a constant $C_{1}>0$ such that

$$
\int_{H}\left|\partial_{x}^{\gamma} \mathcal{D}_{t}^{\nu} u(x, t)\right|^{p} d \mu(x, t) \leq C_{1} \int_{H}\left|\mathcal{D}_{t}^{\rho} u(x, t)\right|^{p} t^{\sigma} d V(x, t)
$$

for all $u \in \boldsymbol{b}_{\alpha}^{p}(\lambda)$.

(2) For $0<\delta<1$, there exists a constant $C_{2}>0$ such that

$$
\mu\left(S_{\delta}^{(\alpha)}(x, t)\right) \leq C_{2} t^{\frac{n}{2 \alpha}+\sigma+1+\left(\frac{|\gamma|}{2 \alpha}+\nu-\rho\right) p}
$$

for all $(x, t) \in H$. 
(3) There exists a constant $C_{3}>0$ such that

$$
\mu\left(Q^{(\alpha)}(x, t)\right) \leq C_{3} t^{\frac{n}{2 \alpha}+\sigma+1+\left(\frac{|\gamma|}{2 \alpha}+\nu-\rho\right) p}
$$

for all $(x, t) \in H$.

Proof. $(1) \Rightarrow(3)$. We assume that $\mu$ satisfies (3.3) of (1). Let $\kappa$ be a real number such that

$$
\kappa>\max \left\{\frac{\lambda+1}{p},\left(\frac{n}{2 \alpha}+\sigma+1\right) \frac{1}{p}-\frac{n+|\gamma|}{2 \alpha}-\rho\right\} .
$$

Let $(y, s) \in H$ be fixed. And we put $u(x, t)=\partial_{x}^{\gamma} \mathcal{D}_{t}^{\kappa} W^{(\alpha)}(x-y, t+s)$ for all $(x, t) \in H$. Then, Lemma 2.1 and Corollary 3.2 imply that $u \in \boldsymbol{b}_{\alpha}^{p}(\lambda)$. Also by Lemma 2.3, there exist constants $\tau, C>0$ such that

$$
\left|\partial_{x}^{\gamma} \mathcal{D}_{t}^{\nu} u(x, t)\right|=\left|\partial_{x}^{2 \gamma} \mathcal{D}_{t}^{\nu+\kappa} W^{(\alpha)}(x-y, t+s)\right| \geq C s^{-\frac{n+2|\gamma|}{2 \alpha}-\nu-\kappa}
$$

for all $(x, t) \in Q^{(\alpha)}(y, \tau s)$. Therefore, we obtain

$$
\begin{aligned}
\int_{H}\left|\partial_{x}^{\gamma} \mathcal{D}_{t}^{\nu} u(x, t)\right|^{p} d \mu(x, t) & \geq \int_{Q^{(\alpha)}(y, \tau s)}\left|\partial_{x}^{2 \gamma} \mathcal{D}_{t}^{\nu+\kappa} W^{(\alpha)}(x-y, t+s)\right|^{p} d \mu(x, t) \\
& \geq C s^{-\left(\frac{n+2|\gamma|}{2 \alpha}+\nu+\kappa\right) p} \int_{Q^{(\alpha)}(y, \tau s)} d \mu(x, t) \\
& =C s^{-\left(\frac{n+2|\gamma|}{2 \alpha}+\nu+\kappa\right) p} \mu\left(Q^{(\alpha)}(y, \tau s)\right) .
\end{aligned}
$$

Moreover, since $\sigma>-1$ and $\kappa>\left(\frac{n}{2 \alpha}+\sigma+1\right) \frac{1}{p}-\frac{n+|\gamma|}{2 \alpha}-\rho$, Lemma 2.1 and Corollary 3.2 imply that

$$
\int_{H}\left|\partial_{x}^{\gamma} \mathcal{D}_{t}^{\rho+\kappa} W^{(\alpha)}(x-y, t+s)\right|^{p} t^{\sigma} d V(x, t) \leq C s^{\frac{n}{2 \alpha}+\sigma+1-\left(\frac{n+|\gamma|}{2 \alpha}+\rho+\kappa\right) p}
$$

for all $(y, s) \in H$. Hence by (3.4) and (3.5), we obtain

$$
\mu\left(Q^{(\alpha)}(y, \tau s)\right) \leq C s^{\frac{n}{2 \alpha}+\sigma+1+\left(\frac{|\gamma|}{2 \alpha}+\nu-\rho\right) p}
$$

for all $(y, s) \in H$. Since $s$ is arbitrary, we can get the inequality of (3).

$(3) \Rightarrow(1)$. We assume that $\mu$ satisfies the inequality of (3). It suffices to show that the conditions of Lemma 3.3 are satisfied. We put $\varepsilon=\frac{n}{2 \alpha}+\sigma+1+$ $\left(\frac{|\gamma|}{2 \alpha}+\nu-\rho\right) p$. Furthermore, let $\eta$ and $\kappa$ be real numbers which satisfy

$$
-\left(\frac{|\gamma|}{2 \alpha}+\nu-\rho\right) p<\eta<\sigma+1 \text { and } \kappa>\max \left\{\frac{\lambda+1}{p}, \sigma+1-\rho-\frac{\eta(p-1)}{p}\right\} \text {. }
$$

Also, we put

$$
\theta=-\left(\frac{|\gamma|}{2 \alpha}+\nu-\rho+\frac{\eta}{p}\right)(p-1)
$$

Then, we have

$$
\theta+\varepsilon=\frac{n}{2 \alpha}+\sigma+1+\frac{|\gamma|}{2 \alpha}+\nu-\rho-\frac{\eta(p-1)}{p}>\frac{n}{2 \alpha}+\sigma+1-\eta>\frac{n}{2 \alpha}
$$


and

$$
\theta+\varepsilon=\frac{n}{2 \alpha}+\sigma+1+\frac{|\gamma|}{2 \alpha}+\nu-\rho-\frac{\eta(p-1)}{p}<\frac{n+|\gamma|}{2 \alpha}+\nu+\kappa
$$

Therefore by (1) of Lemma 2.1 and Lemma 3.1, we obtain

$$
\begin{aligned}
& \int_{H}\left|\partial_{x}^{\gamma} \mathcal{D}_{t}^{\nu+\kappa} W^{(\alpha)}(x-y, t+s)\right| t^{-\left(\frac{|\gamma|}{2 \alpha}+\nu-\rho+\frac{\eta}{p}\right)(p-1)} d \mu(x, t) \\
\leq & C s^{-\left(\rho+\kappa-\sigma-1+\frac{\eta(p-1)}{p}\right)}
\end{aligned}
$$

for all $(y, s) \in H$. Also, since $-\frac{|\gamma|}{2 \alpha}-\nu<\frac{\eta}{p}-\rho<\kappa$, the conditions of Lemma 3.3 are satisfied. Therefore, we can get the inequality (3.3) of (1).

The equivalence $(2) \Leftrightarrow(3)$ immediately follows from Lemma 2.2. Thus, this completes the proof.

\section{Boundedness of the representing operator}

In this section, we study the boundedness of the representing operator $U_{p, \mathbb{X}}^{\kappa}$ defined in Section 1. In Theorem 4.3 below, we give the proof of Theorem 1. First, we introduce the following operator, which is used in our argument. Let $0<\alpha \leq 1,1 \leq p<\infty$, and $\lambda>-1$. Furthermore, let $\gamma \in \mathbb{N}_{0}^{n}, \nu \in \mathbb{R}$, and $\mathbb{X}=\left\{\left(\bar{x}_{j}, t_{j}\right)\right\}$ be a sequence in $H$. For a function $u$ on $H$, the operator $T_{p, \mathbb{X}}^{\gamma, \nu}$ is defined by

$$
T_{p, \mathbb{X}}^{\gamma, \nu} u=\left\{t_{j}^{\left(\frac{n}{2 \alpha}+\lambda+1\right) \frac{1}{p}+\frac{|\gamma|}{2 \alpha}+\nu} \partial_{x}^{\gamma} \mathcal{D}_{t}^{\nu} u\left(x_{j}, t_{j}\right)\right\} .
$$

The following lemma is necessary and sufficient conditions for the operator $T_{p, \mathbb{X}}^{\gamma, \nu}$ to be a bounded operator from $\boldsymbol{b}_{\alpha}^{p}(\lambda)$ to $\ell^{p}$. For any finite set $E \subset H$, we denote by $\#(E)$ the number of points in $E$.

Lemma 4.1. Let $0<\alpha \leq 1,1 \leq p<\infty$, and $\lambda>-1$. Furthermore, let $\gamma \in \mathbb{N}_{0}^{n}, \nu>-\frac{\lambda+1}{p}$, and $\mathbb{X}=\left\{\left(x_{j}, t_{j}\right)\right\}$ be a sequence in $H$. Then, the following statements are equivalent:

(1) $T_{p, \mathbb{X}}^{\gamma, \nu}: \boldsymbol{b}_{\alpha}^{p}(\lambda) \rightarrow \ell^{p}$ is bounded.

(2) There exist $L \in \mathbb{N}$ and $0<\delta<1$ such that $\#\left(\mathbb{X} \cap S_{\delta}^{(\alpha)}(x, t)\right) \leq L$ for all $(x, t) \in H$.

(3) There exists $M \in \mathbb{N}$ such that $\#\left(\mathbb{X} \cap Q^{(\alpha)}(x, t)\right) \leq M$ for all $(x, t) \in H$.

(4) For any $0<\varepsilon<1$, there exists $K \in \mathbb{N}$ such that $\mathbb{X}=\mathbb{X}_{1} \cup \cdots \cup \mathbb{X}_{K}$ and each sequence $\mathbb{X}_{i}$ is $\varepsilon$-separated in the $\alpha$-parabolic sense.

Proof. (1) $\Leftrightarrow(2)$. Let $u \in \boldsymbol{b}_{\alpha}^{p}(\lambda)$ and $\mathbb{X}=\left\{X_{j}\right\}=\left\{\left(x_{j}, t_{j}\right)\right\}$ be a sequence in $H$. Also, let $\mu=\sum_{j} t_{j}^{\frac{n}{2 \alpha}+\lambda+1+\left(\frac{|\gamma|}{2 \alpha}+\nu\right) p} \delta_{X_{j}}$, where $\delta_{X_{j}}$ denotes a Dirac measure at the point $X_{j}$. Then we have

$\left\|T_{p, \mathbb{X}}^{\gamma, \nu} u\right\|_{\ell^{p}}^{p}=\sum_{j} t_{j}^{\frac{n}{2 \alpha}+\lambda+1+\left(\frac{|\gamma|}{2 \alpha}+\nu\right) p}\left|\partial_{x}^{\gamma} \mathcal{D}_{t}^{\nu} u\left(x_{j}, t_{j}\right)\right|^{p}=\int_{H}\left|\partial_{x}^{\gamma} \mathcal{D}_{t}^{\nu} u(x, t)\right|^{p} d \mu(x, t)$. 
Let $(y, s) \in H$ be fixed. If $\left(x_{j}, t_{j}\right) \in S_{\delta}^{(\alpha)}(y, s)$, then we have $\frac{1-\delta}{1+\delta} s<t_{j}<\frac{1+\delta}{1-\delta} s$. Therefore, we obtain

$$
\begin{aligned}
\mu\left(S_{\delta}^{(\alpha)}(y, s)\right) & =\sum_{j} t_{j}^{\frac{n}{2 \alpha}+\lambda+1+\left(\frac{|\gamma|}{2 \alpha}+\nu\right) p} \delta_{X_{j}}\left(S_{\delta}^{(\alpha)}(y, s)\right) \\
& \approx s^{\frac{n}{2 \alpha}+\lambda+1+\left(\frac{|\gamma|}{2 \alpha}+\nu\right) p} \#\left(\mathbb{X} \cap S_{\delta}^{(\alpha)}(y, s)\right),
\end{aligned}
$$

where $A \approx B$ means $C^{-1} A \leq B \leq C A$ for some $C>0$. Hence the equivalence $(1) \Leftrightarrow(2)$ immediately follows from Theorem 3.4.

The equivalence $(2) \Leftrightarrow(3) \Leftrightarrow(4)$ is already given in Theorem 1 of [7]. Thus, this completes the proof.

In order to prove the main theorem of this section, we also give the following lemma.

Lemma 4.2. Let $0<\alpha \leq 1$. For every $\theta>-1$ and $c>0$, there exists a constant $C>0$ such that

$$
\frac{s^{\theta}}{\left(t+s+|x-y|^{2 \alpha}\right)^{c}} \leq \frac{C F(\delta)}{s^{\frac{n}{2 \alpha}+1}} \int_{S_{\delta}^{(\alpha)}(y, s)} \frac{r^{\theta}}{\left(t+r+|x-z|^{2 \alpha}\right)^{c}} d V(z, r)
$$

for all $0<\delta<1$ and $(x, t),(y, s) \in H$, where

$$
F(\delta)=\frac{\left(1-\delta^{2}\right)^{\frac{n}{2 \alpha}}+\theta+1-c}{\delta^{\frac{n}{2 \alpha}}\left\{\left(1+\delta^{2}\right)^{2(\theta+1)}-\left(1-\delta^{2}\right)^{2(\theta+1)}\right\}} .
$$

Proof. Let $\theta>-1$ and $c>0$. We consider the following:

$$
\begin{aligned}
& \frac{s^{\theta}}{\left(t+s+|x-y|^{2 \alpha}\right)^{c}} \\
= & \frac{s^{\theta}}{\left(t+s+|x-y|^{2 \alpha}\right)^{c}} \int_{S_{\delta}^{(\alpha)}(y, s)} r^{\theta} d V(z, r)\left(\int_{S_{\delta}^{(\alpha)}(y, s)} r^{\theta} d V(z, r)\right)^{-1} \\
= & s^{\theta} \int_{S_{\delta}^{(\alpha)}(y, s)} \frac{r^{\theta}}{\left(t+s+|x-y|^{2 \alpha}\right)^{c}} d V(z, r)\left(\int_{S_{\delta}^{(\alpha)}(y, s)} r^{\theta} d V(z, r)\right)^{-1} .
\end{aligned}
$$

We note that

$$
\begin{aligned}
& \int_{S_{\delta}^{(\alpha)}(y, s)} r^{\theta} d V(z, r) \\
= & B_{n}\left(\frac{2 \delta}{1-\delta^{2}} s\right)^{\frac{n}{2 \alpha}} \frac{1}{\theta+1}\left\{\left(\frac{1+\delta}{1-\delta} s\right)^{\theta+1}-\left(\frac{1-\delta}{1+\delta} s\right)^{\theta+1}\right\} \\
= & \frac{2^{\frac{n}{2 \alpha}} B_{n}}{\theta+1} \cdot \frac{\delta^{\frac{n}{2 \alpha}}\left\{(1+\delta)^{2(\theta+1)}-(1-\delta)^{2(\theta+1)}\right\}}{\left(1-\delta^{2}\right)^{\frac{n}{2 \alpha}+\theta+1}} s^{\frac{n}{2 \alpha}+\theta+1},
\end{aligned}
$$


where $B_{n}$ is the volume of the unit ball in $\mathbb{R}^{n}$. Moreover, if $(z, r) \in S_{\delta}^{(\alpha)}(y, s)$, then we have

$$
\begin{aligned}
& t+r+|x-z|^{2 \alpha} \leq t+r+\{|x-y|+|y-z|\}^{2 \alpha} \\
\leq & t+\frac{1+\delta}{1-\delta} s+\left\{|x-y|+\left(\frac{2 \delta}{1-\delta^{2}} s\right)^{\frac{1}{2 \alpha}}\right\}^{2 \alpha} \\
\leq & t+\left(\frac{1+\delta}{1-\delta}+2^{2 \alpha} \frac{2 \delta}{1-\delta^{2}}\right) s+2^{2 \alpha}|x-y|^{2 \alpha} \\
\leq & \frac{12}{1-\delta^{2}}\left(t+s+|x-y|^{2 \alpha}\right) .
\end{aligned}
$$

Hence, we obtain

$$
\begin{aligned}
& \int_{S_{\delta}^{(\alpha)}(y, s)} \frac{r^{\theta}}{\left(t+r+|x-z|^{2 \alpha}\right)^{c}} d V(z, r) \\
\geq & \frac{2^{\frac{n}{2 \alpha}} B_{n}}{12^{c}(\theta+1)} \cdot \frac{\delta^{\frac{n}{2 \alpha}}\left\{(1+\delta)^{2(\theta+1)}-(1-\delta)^{2(\theta+1)}\right\}}{\left(1-\delta^{2}\right)^{\frac{n}{2 \alpha}+\theta+1-c}} \cdot \frac{s^{\theta}}{\left(t+s+|x-y|^{2 \alpha}\right)^{c}} .
\end{aligned}
$$

This completes the proof.

We extend the definition of the representing operator $U_{p, \mathbb{X}}^{\kappa}$ in Section 1. Let $0<\alpha \leq 1,1 \leq p<\infty$, and $\lambda>-1$. Furthermore, let $\beta \in \mathbb{N}_{0}^{n}, \kappa \in \mathbb{R}$, and $\mathbb{X}=\left\{\left(x_{j}, t_{j}\right)\right\}$ be a sequence in $H$. For a sequence of real numbers $\left\{\eta_{j}\right\}$, we define a representing operator $U_{p, \mathbb{X}}^{\beta, \kappa}$ by

$$
\begin{aligned}
& U_{p, \mathbb{X}}^{\beta, \kappa}\left(\left\{\eta_{j}\right\}\right)(x, t) \\
= & \sum_{j} \eta_{j} t_{j}^{\frac{n+|\beta|}{2 \alpha}+\kappa-\left(\frac{n}{2 \alpha}+\lambda+1\right) \frac{1}{p}} \partial_{x}^{\beta} \mathcal{D}_{t}^{\kappa} W^{(\alpha)}\left(x-x_{j}, t+t_{j}\right), \quad(x, t) \in H .
\end{aligned}
$$

The following theorem gives necessary and sufficient conditions for the representing operator $U_{p, \mathbb{X}}^{\beta, \kappa}$ to be bounded from $\ell^{p}$ to $\boldsymbol{b}_{\alpha}^{p}(\lambda)$. We note that Theorem 4.3 contains the result of Theorem 1 .

Theorem 4.3. Let $0<\alpha \leq 1,1<p<\infty$, and $\lambda>-1$. Furthermore, let $\beta \in \mathbb{N}_{0}^{n}, \kappa>\frac{\lambda+1}{p}$, and $\mathbb{X}=\left\{\left(x_{j}, t_{j}\right)\right\}$ be a sequence in $H$. Then, the following statements are equivalent:

(1) $U_{p, \mathbb{X}}^{\beta, \kappa}: \ell^{p} \rightarrow \boldsymbol{b}_{\alpha}^{p}(\lambda)$ is bounded.

(2) For any $0<\varepsilon<1$, there exist $K \in \mathbb{N}$ such that $\mathbb{X}=\mathbb{X}_{1} \cup \cdots \cup \mathbb{X}_{K}$ and each sequence $\mathbb{X}_{i}$ is $\varepsilon$-separated in the $\alpha$-parabolic sense.

Moreover, $(2) \Rightarrow(1)$ holds when $p=1$.

Proof. $(1) \Rightarrow(2)$. We assume that $U_{p, \mathbb{X}}^{\beta, \kappa}$ is the bounded operator from $\ell^{p}$ to $\boldsymbol{b}_{\alpha}^{p}(\lambda)$. Then, there exists the adjoint operator $\left(U_{p, \mathbb{X}}^{\beta, \kappa}\right)^{*}$ of $U_{p, \mathbb{X}}^{\beta, \kappa}$ such that $\left(U_{p, \mathbb{X}}^{\beta, \kappa}\right)^{*}:\left(\boldsymbol{b}_{\alpha}^{p}(\lambda)\right)^{*} \rightarrow\left(\ell^{p}\right)^{*}$ is bounded. By Lemma 2.6, $\left(U_{p, \mathbb{X}}^{\beta, \kappa}\right)^{*}: \boldsymbol{b}_{\alpha}^{q}(\lambda) \rightarrow \ell^{q}$ is bounded, where $q$ is the exponent conjugate to $p$. Let $\left\{e_{j}\right\}$ be the standard 
basis of $\ell^{p}$. Furthermore, let $(\cdot, \cdot)$ be the usual pairing of $\ell^{p}$ and $\ell^{q}$, and recall that $\langle\cdot, \cdot\rangle_{\lambda}$ is the integral pairing of $\boldsymbol{b}_{\alpha}^{p}(\lambda)$ and $\boldsymbol{b}_{\alpha}^{q}(\lambda)$ defined in Lemma 2.6. For $u \in \boldsymbol{b}_{\alpha}^{q}(\lambda)$, we have

$$
\begin{aligned}
& \left(\left(U_{p, \mathbb{X}}^{\beta, \kappa}\right)^{*} u, e_{j}\right) \\
= & \left\langle u, U_{p, \mathbb{X}}^{\beta, \kappa}\left(e_{j}\right)\right\rangle_{\lambda} \\
= & \int_{H} u(y, s) t_{j}^{\frac{n+|\beta|}{2 \alpha}+\kappa-\left(\frac{n}{2 \alpha}+\lambda+1\right) \frac{1}{p}} \partial_{x}^{\beta} \mathcal{D}_{t}^{\kappa} W^{(\alpha)}\left(y-x_{j}, s+t_{j}\right) s^{\lambda} d V(y, s) \\
= & t_{j}^{\frac{n+|\beta|}{2 \alpha}+\kappa-\left(\frac{n}{2 \alpha}+\lambda+1\right) \frac{1}{p}} \int_{H} u(y, s) \partial_{x}^{\beta} \mathcal{D}_{t}^{\kappa} W^{(\alpha)}\left(y-x_{j}, s+t_{j}\right) s^{\lambda} d V(y, s) .
\end{aligned}
$$

By a change of variable $y=2 x_{j}-z$, we have

$$
\begin{aligned}
& \left(\left(U_{p, \mathbb{X}}^{\beta, \kappa}\right)^{*} u, e_{j}\right) \\
= & t_{j}^{\frac{n+|\beta|}{2 \alpha}+\kappa-\left(\frac{n}{2 \alpha}+\lambda+1\right) \frac{1}{p}} \int_{H} v(z, s) \partial_{x}^{\beta} \mathcal{D}_{t}^{\kappa} W^{(\alpha)}\left(x_{j}-z, s+t_{j}\right) s^{\lambda} d V(z, s),
\end{aligned}
$$

where $v(z, s)=u\left(2 x_{j}-z, s\right)$. Differentiating through the integral, Lemma 2.5 implies that

$$
\begin{aligned}
& C_{\lambda+1} \int_{H} v(z, s) \partial_{x}^{\beta} \mathcal{D}_{t}^{\kappa} W^{(\alpha)}\left(x_{j}-z, s+t_{j}\right) s^{\lambda} d V(z, s) \\
= & \partial_{x}^{\beta} \mathcal{D}_{t}^{\kappa-(\lambda+1)}\left(v\left(x_{j}, t_{j}\right)\right)=(-1)^{|\beta|} \partial_{x}^{\beta} \mathcal{D}_{t}^{\kappa-(\lambda+1)} u\left(x_{j}, t_{j}\right) .
\end{aligned}
$$

Therefore, we obtain

$$
\begin{aligned}
\left(\left(U_{p, \mathbb{X}}^{\beta, \kappa}\right)^{*} u, e_{j}\right) & =\frac{(-1)^{|\beta|}}{C_{\lambda+1}} t_{j}^{\frac{n+|\beta|}{2 \alpha}+\kappa-\left(\frac{n}{2 \alpha}+\lambda+1\right) \frac{1}{p}} \partial_{x}^{\beta} \mathcal{D}_{t}^{\kappa-(\lambda+1)} u\left(x_{j}, t_{j}\right) \\
& =\frac{(-1)^{|\beta|}}{C_{\lambda+1}} t_{j}^{\left(\frac{n}{2 \alpha}+\lambda+1\right) \frac{1}{q}+\frac{|\beta|}{2 \alpha}+\kappa-(\lambda+1)} \partial_{x}^{\beta} \mathcal{D}_{t}^{\kappa-(\lambda+1)} u\left(x_{j}, t_{j}\right) \\
& =\left(\frac{(-1)^{|\beta|}}{C_{\lambda+1}} T_{q, \mathbb{X}}^{\beta, \kappa-(\lambda+1)} u, e_{j}\right) .
\end{aligned}
$$

Hence we have

$$
\left(U_{p, \mathbb{X}}^{\beta, \kappa}\right)^{*} u=\frac{(-1)^{|\beta|}}{C_{\lambda+1}} T_{q, \mathbb{X}}^{\beta, \kappa-(\lambda+1)} u
$$

for all $u \in \boldsymbol{b}_{\alpha}^{q}(\lambda)$. Since $\left(U_{p, \mathbb{X}}^{\beta, \kappa}\right)^{*}: \boldsymbol{b}_{\alpha}^{q}(\lambda) \rightarrow \ell^{q}$ is bounded, so is $T_{q, \mathbb{X}}^{\beta, \kappa-(\lambda+1)}$. Therefore by Lemma 4.1 , we obtain the implication $(1) \Rightarrow(2)$.

$(2) \Rightarrow(1)$. We may assume that the sequence $\mathbb{X}$ in $H$ is $\delta$-separated in the $\alpha$-parabolic sense. Let $\left\{\eta_{j}\right\} \in \ell^{p}$ and $(x, t) \in H$. By Lemmas 2.1 and 4.2 , we 
have

$$
\begin{aligned}
& \left|U_{p, \mathbb{X}}^{\beta, \kappa}\left(\left\{\eta_{j}\right\}\right)(x, t)\right| \\
\leq & \sum_{j}\left|\eta_{j}\right| t_{j}^{\frac{n+|\beta|}{2 \alpha}+\kappa-\left(\frac{n}{2 \alpha}+\lambda+1\right) \frac{1}{p}}\left|\partial_{x}^{\beta} \mathcal{D}_{t}^{\kappa} W^{(\alpha)}\left(x-x_{j}, t+t_{j}\right)\right| \\
\leq & C \sum_{j}\left|\eta_{j}\right| t_{j}^{-\frac{n}{2 \alpha}-1} \int_{S_{\delta}^{(\alpha)}\left(x_{j}, t_{j}\right)} \frac{r^{\frac{n+|\beta|}{2 \alpha}+\kappa-\left(\frac{n}{2 \alpha}+\lambda+1\right) \frac{1}{p}}}{\left(t+r+|x-z|^{2 \alpha}\right)^{\frac{n+|\beta|}{2 \alpha}+\kappa}} d V(z, r) \\
= & \int_{H} \frac{f(z, r) r^{\frac{n+|\beta|}{2 \alpha}+\kappa-\left(\frac{n}{2 \alpha}+\lambda+1\right) \frac{1}{p}}}{\left(t+r+|x-z|^{2 \alpha}\right)^{\frac{n+|\beta|}{2 \alpha}}+\kappa} d V(z, r),
\end{aligned}
$$

where

$$
f(z, r)=C \sum_{j}\left|\eta_{j}\right| t_{j}^{-\frac{n}{2 \alpha}-1} \chi_{j}(z, r)
$$

and $\chi_{j}$ is the characteristic function of $S_{\delta}^{(\alpha)}\left(x_{j}, t_{j}\right)$.

First, we show that $U_{p, \mathbb{X}}^{\beta, \kappa}: \ell^{p} \rightarrow L^{p}(\lambda)$ is bounded when $1<p<\infty$. Let $q$ be the exponent conjugate to $p$. And we take a real number $c$ such that

$$
\begin{aligned}
\frac{n}{2 \alpha}+1+\left(\frac{|\beta|}{2 \alpha}+\kappa-\lambda-1\right) \frac{q}{p} & <\frac{c}{p}<\frac{n}{2 \alpha}+\lambda+2+\left(\frac{|\beta|}{2 \alpha}+\kappa-\lambda-1\right) q, \\
\left(\frac{n}{2 \alpha}+1\right) \frac{p}{q} & <\frac{c}{q}<\left(\frac{n}{2 \alpha}+1\right) \frac{p}{q}+\frac{|\beta|}{2 \alpha}+\kappa .
\end{aligned}
$$

By the Hölder inequality, we obtain

$$
\begin{aligned}
\left|U_{p, \mathbb{X}}^{\beta, \kappa}\left(\left\{\eta_{j}\right\}\right)(x, t)\right|^{p} \leq & \left(\int_{H} \frac{f(z, r) r^{\frac{n+|\beta|}{2 \alpha}+\kappa-\left(\frac{n}{2 \alpha}+\lambda+1\right) \frac{1}{p}} r^{\frac{c}{p q}} r^{-\frac{c}{p q}}}{\left(t+r+|x-z|^{2 \alpha}\right)^{\frac{n+|\beta|}{2 \alpha}}+\kappa} d V(z, r)\right)^{p} \\
\leq & C \int_{H} \frac{f(z, r)^{p} r^{\frac{c}{q}}}{\left(t+r+|x-z|^{2 \alpha}\right)^{\frac{n+|\beta|}{2 \alpha}+\kappa}} d V(z, r) \\
& \times\left(\int_{H} \frac{r^{\left(\frac{n+|\beta|}{2 \alpha}+\kappa\right) q-\left(\frac{n}{2 \alpha}+\lambda+1\right) \frac{q}{p}-\frac{c}{p}}}{\left(t+r+|x-z|^{2 \alpha}\right)^{\frac{n+|\beta|}{2 \alpha}+\kappa}} d V(z, r)\right)^{\frac{p}{q}}
\end{aligned}
$$

Since $c$ satisfies the condition (4.2), Corollary 3.2 implies that (4.5)

$$
\left(\int_{H} \frac{r^{\left(\frac{n+|\beta|}{2 \alpha}+\kappa\right) q-\left(\frac{n}{2 \alpha}+\lambda+1\right) \frac{q}{p}-\frac{c}{p}}}{\left(t+r+|x-z|^{2 \alpha}\right)^{\frac{n+|\beta|}{2 \alpha}+\kappa}} d V(z, r)\right)^{\frac{p}{q}} \leq C t^{\left(\frac{n}{2 \alpha}+1\right) \frac{p}{q}+\frac{|\beta|}{2 \alpha}+\kappa-\lambda-1-\frac{c}{q}} .
$$


Hence by (4.4), (4.5), and the Fubini theorem, we have

$$
\begin{aligned}
& \left\|U_{p, \mathbb{X}}^{\beta, \kappa}\left(\left\{\eta_{j}\right\}\right)\right\|_{L^{p}(\lambda)}^{p} \\
= & \int_{H}\left|U_{p, \mathbb{X}}^{\beta, \kappa}\left(\left\{\eta_{j}\right\}\right)(x, t)\right|^{p} t^{\lambda} d V(x, t) \\
\leq & C \int_{H} \int_{H} \frac{f(z, r)^{p} r^{\frac{c}{q}}}{\left(t+r+|x-z|^{2 \alpha}\right)^{\frac{n+|\beta|}{2 \alpha}+\kappa}} d V(z, r) t^{\left(\frac{n}{2 \alpha}+1\right) \frac{p}{q}+\frac{|\beta|}{2 \alpha}+\kappa-1-\frac{c}{q}} d V(x, t) \\
\leq & C \int_{H} f(z, r)^{p} r^{\frac{c}{q}} \int_{H} \frac{t^{\left(\frac{n}{2 \alpha}+1\right) \frac{p}{q}+\frac{|\beta|}{2 \alpha}+\kappa-1-\frac{c}{q}}}{\left(t+r+|x-z|^{2 \alpha}\right)^{\frac{n+|\beta|}{2 \alpha}+\kappa}} d V(x, t) d V(z, r) .
\end{aligned}
$$

Since $c$ satisfies the condition (4.3), Corollary 3.2 implies that

$$
\int_{H} \frac{t^{\left(\frac{n}{2 \alpha}+1\right) \frac{p}{q}+\frac{|\beta|}{2 \alpha}+\kappa-1-\frac{c}{q}}}{\left(t+r+|x-z|^{2 \alpha}\right)^{\frac{n+|\beta|}{2 \alpha}+\kappa}} d V(x, t) \leq C r^{\left(\frac{n}{2 \alpha}+1\right) \frac{p}{q}-\frac{c}{q}}=C r^{\left(\frac{n}{2 \alpha}+1\right)(p-1)-\frac{c}{q}} .
$$

Therefore, we obtain

$$
\left\|U_{p, \mathbb{X}}^{\beta, \kappa}\left(\left\{\eta_{j}\right\}\right)\right\|_{L^{p}(\lambda)}^{p} \leq C \int_{H} f(z, r)^{p} r^{\left(\frac{n}{2 \alpha}+1\right)(p-1)} d V(z, r) .
$$

Since $f(z, r)^{p} \leq \sum_{j}\left|\eta_{j}\right|^{p} t_{j}^{-\left(\frac{n}{2 \alpha}+1\right) p} \chi_{j}(z, r)$, we have

$$
\begin{aligned}
& \int_{H} f(z, r)^{p} r^{\left(\frac{n}{2 \alpha}+1\right)(p-1)} d V(z, r) \\
\leq & C \sum_{j}\left|\eta_{j}\right|^{p} t_{j}^{-\left(\frac{n}{2 \alpha}+1\right) p} \int_{S_{\delta}^{(\alpha)}\left(x_{j}, t_{j}\right)} r^{\left(\frac{n}{2 \alpha}+1\right)(p-1)} d V(z, r) \\
\leq & C \sum_{j}\left|\eta_{j}\right|^{p} t_{j}^{-\frac{n}{2 \alpha}-1} V\left(S_{\delta}^{(\alpha)}\left(x_{j}, t_{j}\right)\right) \\
\leq & C \sum_{j}\left|\eta_{j}\right|^{p} .
\end{aligned}
$$

Thus, $U_{p, \mathbb{X}}^{\beta, \kappa}: \ell^{p} \rightarrow L^{p}(\lambda)$ is bounded when $1<p<\infty$.

Next, we show that $U_{1, \mathbb{X}}^{\beta, \kappa}: \ell^{1} \rightarrow L^{1}(\lambda)$ is bounded. By (4.1) and the Fubini theorem, we have

$$
\begin{aligned}
& \left\|U_{1, \mathbb{X}}^{\beta, \kappa}\left(\left\{\eta_{j}\right\}\right)\right\|_{L^{1}(\lambda)} \\
\leq & C \int_{H} \int_{H} \frac{f(z, r) r^{\frac{|\beta|}{2 \alpha}+\kappa-\lambda-1}}{\left(t+r+|x-z|^{2 \alpha}\right)^{\frac{n+|\beta|}{2 \alpha}+\kappa}} d V(z, r) t^{\lambda} d V(x, t) \\
= & C \int_{H} f(z, r) r^{\frac{|\beta|}{2 \alpha}+\kappa-\lambda-1} \int_{H} \frac{t^{\lambda}}{\left(t+r+|x-z|^{2 \alpha}\right)^{\frac{n+|\beta|}{2 \alpha}+\kappa}} d V(x, t) d V(z, r) .
\end{aligned}
$$


Corollary 3.2 implies that

$$
\int_{H} \frac{t^{\lambda}}{\left(t+r+|x-z|^{2 \alpha}\right)^{\frac{n+|\beta|}{2 \alpha}+\kappa}} d V(x, t) \leq C r^{-\left(\frac{|\beta|}{2 \alpha}+\kappa-\lambda-1\right)} .
$$

Since $f(z, r)=\sum_{j}\left|\eta_{j}\right| t_{j}^{-\frac{n}{2 \alpha}-1} \chi_{j}(z, r)$, we obtain

$$
\begin{aligned}
\left\|U_{1, \mathbb{X}}^{\beta, \kappa}\left(\left\{\eta_{j}\right\}\right)\right\|_{L^{1}(\lambda)} & \leq C \int_{H} f(z, r) d V(z, r) \\
& =C \sum_{j}\left|\eta_{j}\right| t_{j}^{-\frac{n}{2 \alpha}-1} V\left(S_{\delta}^{(\alpha)}\left(x_{j}, t_{j}\right)\right) \leq C \sum_{j}\left|\eta_{j}\right| .
\end{aligned}
$$

Thus, $U_{1, \mathbb{X}}^{\beta, \kappa}: \ell^{1} \rightarrow L^{1}(\lambda)$ is bounded.

Finally, we show that $U_{p, \mathbb{X}}^{\beta, \kappa}\left(\left\{\eta_{j}\right\}\right)$ is $L^{(\alpha)}$-harmonic on $H$. For $0<t_{1}<t_{2}<$ $\infty$, the boundedness of $U_{p, \mathbb{X}}^{\beta, \kappa}$ implies that $U_{p, \mathbb{X}}^{\beta, \kappa}\left(\left\{\eta_{j}\right\}\right)$ satisfies the integrability condition (2.2) for all $\left\{\eta_{j}\right\} \in \ell^{p}$. Moreover, the norm convergence of $U_{p, \mathbb{X}}^{\beta, \kappa}\left(\left\{\eta_{j}\right\}\right)$ implies that $U_{p, \mathbb{X}}^{\beta, \kappa}\left(\left\{\eta_{j}\right\}\right)$ converges uniformly on $\mathbb{R}^{n} \times[\tau, \infty)$ for every $\tau>0$. Therefore by (3) of Lemma 2.1, $U_{p, \mathbb{X}}^{\beta, \kappa}\left(\left\{\eta_{j}\right\}\right)$ is $L^{(\alpha)}$-harmonic on $H$. Thus, we can get the statement (1) when $1 \leq p<\infty$. This completes the proof.

\section{Representing sequences on parabolic Bergman spaces}

In this section, we study representing sequences on $\boldsymbol{b}_{\alpha}^{p}(\lambda)$. In Theorem 5.3 below, we give the proof of Theorem 2. First, we present the following lemma, which is used in the proof of Lemma 5.2 below.

Lemma 5.1 (Lemma 3.1 of [6]). Let $0<\alpha \leq 1$ and $\sigma>0$. Suppose $0<\delta \leq$ $1 / 3$. Then, there exists a constant $C=C(\alpha, \sigma)>0$ independent of $\delta$ with the following property: For every $a>0, s>0$, and $\xi, t>0$ with $\xi<\left(\frac{2 \delta}{1-\delta^{2}}\right)^{\frac{1}{2 \alpha}} t$, we have

$$
\int_{0}^{1} \frac{\xi}{(t+s+|a-\xi \tau|)^{\sigma+1}} d \tau \leq C \frac{\delta^{\frac{1}{2 \alpha}}}{(t+s+a)^{\sigma}} .
$$

The following lemma is useful for the proof of Theorem 5.3.

Lemma 5.2. Let $0<\alpha \leq 1, \beta \in \mathbb{N}_{0}^{n}, \kappa>-\frac{n}{2 \alpha}$, and $\theta$ be a real number. Then, there exists a constant $C=C(n, \alpha, \beta, \kappa, \theta)>0$ such that for all $(x, t),(y, s) \in$ $H,(z, r) \in S_{\delta}^{(\alpha)}(y, s)$, and $0<\delta \leq 1 / 3$,

$\left|s^{\theta} \partial_{x}^{\beta} \mathcal{D}_{t}^{\kappa} W^{(\alpha)}(x-y, t+s)-r^{\theta} \partial_{x}^{\beta} \mathcal{D}_{t}^{\kappa} W^{(\alpha)}(x-z, t+r)\right| \leq \frac{C\left(\delta+\delta^{\frac{1}{2 \alpha}}\right) r^{\theta}}{\left(t+r+|x-z|^{2 \alpha}\right)^{\frac{n+|\beta|}{2 \alpha}+\kappa}}$. 
Proof. We have

$$
\begin{gathered}
\quad\left|s^{\theta} \partial_{x}^{\beta} \mathcal{D}_{t}^{\kappa} W^{(\alpha)}(x-y, t+s)-r^{\theta} \partial_{x}^{\beta} \mathcal{D}_{t}^{\kappa} W^{(\alpha)}(x-z, t+r)\right| \\
\leq\left|s^{\theta} \partial_{x}^{\beta} \mathcal{D}_{t}^{\kappa} W^{(\alpha)}(x-y, t+s)-s^{\theta} \partial_{x}^{\beta} \mathcal{D}_{t}^{\kappa} W^{(\alpha)}(x-z, t+s)\right| \\
\quad+\left|s^{\theta} \partial_{x}^{\beta} \mathcal{D}_{t}^{\kappa} W^{(\alpha)}(x-z, t+s)-r^{\theta} \partial_{x}^{\beta} \mathcal{D}_{t}^{\kappa} W^{(\alpha)}(x-z, t+r)\right| .
\end{gathered}
$$

Hence it suffices to show that there exists a constant $C>0$ independent of $\delta$ with the following inequalities

$$
s^{\theta}\left|\partial_{x}^{\beta} \mathcal{D}_{t}^{\kappa} W^{(\alpha)}(x-y, t+s)-\partial_{x}^{\beta} \mathcal{D}_{t}^{\kappa} W^{(\alpha)}(x-z, t+s)\right| \leq \frac{C \delta^{\frac{1}{2 \alpha}} r^{\theta}}{\left(t+r+|x-z|^{2 \alpha}\right)^{\frac{n+|\beta|}{2 \alpha}+\kappa}}
$$

and

$$
\left|s^{\theta} \partial_{x}^{\beta} \mathcal{D}_{t}^{\kappa} W^{(\alpha)}(x-z, t+s)-r^{\theta} \partial_{x}^{\beta} \mathcal{D}_{t}^{\kappa} W^{(\alpha)}(x-z, t+r)\right| \leq \frac{C \delta r^{\theta}}{\left(t+r+|x-z|^{2 \alpha}\right)^{\frac{n+|\beta|}{2 \alpha}+\kappa}} .
$$

We show the inequality (5.2). Since $\frac{1-\delta}{1+\delta} s<r<\frac{1+\delta}{1-\delta} s$ and $0<\delta \leq 1 / 3$, there exists a constant $C>0$ independent of $\delta$ such that $C^{-1} r^{\theta} \leq s^{\theta} \leq C r^{\theta}$. Furthermore, we have

$$
\begin{aligned}
& \left|\partial_{x}^{\beta} \mathcal{D}_{t}^{\kappa} W^{(\alpha)}(x-y, t+s)-\partial_{x}^{\beta} \mathcal{D}_{t}^{\kappa} W^{(\alpha)}(x-z, t+s)\right| \\
\leq & \left|\int_{0}^{1}(z-y) \cdot \nabla_{x} \partial_{x}^{\beta} \mathcal{D}_{t}^{\kappa} W^{(\alpha)}(\tau(z-y)-(z-x), t+s) d \tau\right| \\
\leq & \int_{0}^{1}|z-y| \cdot\left|\nabla_{x} \partial_{x}^{\beta} \mathcal{D}_{t}^{\kappa} W^{(\alpha)}(\tau(z-y)-(z-x), t+s)\right| d \tau .
\end{aligned}
$$

Lemmas 2.1 and 5.1 imply that

$$
\begin{aligned}
& \int_{0}^{1}|z-y| \cdot\left|\nabla_{x} \partial_{x}^{\beta} \mathcal{D}_{t}^{\kappa} W^{(\alpha)}(\tau(z-y)-(z-x), t+s)\right| d \tau \\
\leq & C \int_{0}^{1} \frac{|z-y|}{\left(t+s+|\tau(z-y)-(z-x)|^{2 \alpha}\right)^{\frac{n+|\beta|+1}{2 \alpha}+\kappa}} d \tau \\
\leq & C \int_{0}^{1} \frac{|z-y|}{\left(t^{\frac{1}{2 \alpha}}+s \frac{1}{2 \alpha}+|| z-x|-\tau| z-y||\right)^{n+|\beta|+1+2 \alpha \kappa}} d \tau \\
\leq & \frac{C \delta^{\frac{1}{2 \alpha}}}{\left(t^{\frac{1}{2 \alpha}}+s^{\frac{1}{2 \alpha}}+|x-z|\right)^{n+|\beta|+2 \alpha \kappa}} \leq \frac{C \delta \frac{1}{2 \alpha}}{\left(t+s+|x-z|^{2 \alpha}\right)^{\frac{n+|\beta|}{2 \alpha}+\kappa}} .
\end{aligned}
$$

Since $\frac{1-\delta}{1+\delta} r<s<\frac{1+\delta}{1-\delta} r$ and $0<\delta \leq 1 / 3$, we obtain the inequality (5.2). 
We show the inequality (5.3). Let $\theta \neq 0$. Then, Lemma 2.1 implies that

$$
\begin{aligned}
& \left|s^{\theta} \partial_{x}^{\beta} \mathcal{D}_{t}^{\kappa} W^{(\alpha)}(x-z, t+s)-r^{\theta} \partial_{x}^{\beta} \mathcal{D}_{t}^{\kappa} W^{(\alpha)}(x-z, t+r)\right| \\
= & \left|\int_{r}^{s} \frac{d}{d \tau}\left(\tau^{\theta} \partial_{x}^{\beta} \mathcal{D}_{t}^{\kappa} W^{(\alpha)}(x-z, t+\tau)\right) d \tau\right| \\
\leq & |\theta|\left|\int_{r}^{s} \tau^{\theta-1} \partial_{x}^{\beta} \mathcal{D}_{t}^{\kappa} W^{(\alpha)}(x-z, t+\tau) d \tau\right|+\left|\int_{r}^{s} \tau^{\theta} \partial_{x}^{\beta} \mathcal{D}_{t}^{\kappa+1} W^{(\alpha)}(x-z, t+\tau) d \tau\right| \\
\leq & C\left\{\left|\int_{r}^{s} \frac{\tau^{\theta-1}}{\left(t+\tau+|x-z|^{2 \alpha}\right)^{\frac{n+|\beta|}{2 \alpha}}+\kappa} d \tau\right|+\left|\int_{r}^{s} \frac{\tau^{\theta}}{\left(t+\tau+|x-z|^{2 \alpha}\right)^{\frac{n+|\beta|}{2 \alpha}+\kappa+1}} d \tau\right|\right\} \\
\leq & C\left|\int_{r}^{s} \frac{\tau^{\theta-1}}{\left(t+\tau+|x-z|^{2 \alpha}\right)^{\frac{n+|\beta|}{2 \alpha}+\kappa}} d \tau\right| .
\end{aligned}
$$

Since $\frac{1-\delta}{1+\delta} r<s<\frac{1+\delta}{1-\delta} r$ and $0<\delta \leq 1 / 3$, there exists a constant $C>0$ independent of $\delta$ such that

$$
\begin{aligned}
\left|\int_{r}^{s} \frac{\tau^{\theta-1}}{\left(t+\tau+|x-z|^{2 \alpha}\right)^{\frac{n+|\beta|}{2 \alpha}+\kappa}} d \tau\right| & \leq \frac{C r^{\theta-1}|s-r|}{\left(t+r+|x-z|^{2 \alpha}\right)^{\frac{n+|\beta|}{2 \alpha}+\kappa}} \\
& \leq \frac{C \delta r^{\theta}}{\left(t+r+|x-z|^{2 \alpha}\right)^{\frac{n+|\beta|}{2 \alpha}+\kappa}} .
\end{aligned}
$$

Hence, we obtain the inequality (5.3). When $\theta=0$, we can also show the inequality (5.3), easily. Thus, we obtain the inequality (5.1).

Now, we show the main theorem in this section. Let $0<\delta \leq 1 / 3$. And suppose that $\left\{\left(x_{j}, t_{j}\right)\right\}$ is a $\delta$-lattice in the $\alpha$-parabolic sense, that is, $H=$ $\cup_{j} S_{\delta}^{(\alpha)}\left(x_{j}, t_{j}\right)$ and there exists $0<\varepsilon<\delta$ such that $\left\{\left(x_{j}, t_{j}\right)\right\}$ is $\varepsilon$-separated in the $\alpha$-parabolic sense. Then, we take a pairwise disjoint covering $\left\{S_{j}\right\}$ of $H$ as follows:

$$
\begin{aligned}
& S_{1}=S_{\delta}^{(\alpha)}\left(x_{1}, t_{1}\right) \backslash \bigcup_{k \geq 2} S_{\varepsilon}^{(\alpha)}\left(x_{k}, t_{k}\right), \\
& S_{2}=S_{\delta}^{(\alpha)}\left(x_{2}, t_{2}\right) \backslash\left\{S_{1} \cup\left(\bigcup_{k \geq 3} S_{\varepsilon}^{(\alpha)}\left(x_{k}, t_{k}\right)\right)\right\}, \\
& \cdots, \\
& S_{j}=S_{\delta}^{(\alpha)}\left(x_{j}, t_{j}\right) \backslash\left\{\left(\bigcup_{\ell \leq j-1} S_{\ell}\right) \cup\left(\bigcup_{k \geq j+1} S_{\varepsilon}^{(\alpha)}\left(x_{k}, t_{k}\right)\right)\right\} .
\end{aligned}
$$

It is easy to see that $S_{\varepsilon}^{(\alpha)}\left(x_{j}, t_{j}\right) \subset S_{j} \subset S_{\delta}^{(\alpha)}\left(x_{j}, t_{j}\right) \subseteq S_{1 / 3}^{(\alpha)}\left(x_{j}, t_{j}\right)$ and there exists a constant $C>0$ independent of $\delta$ with $V\left(S_{j}\right) \leq C t_{j}^{\frac{n}{2 \alpha}+1}$ for all $j \geq 1$. We show our $\boldsymbol{b}_{\alpha}^{p}(\lambda)$-representation theorem. 
Theorem 5.3. Let $0<\alpha \leq 1,1 \leq p<\infty, \lambda>-1$, and $\kappa>\frac{\lambda+1}{p}$ be a real number. Then, there exists $0<\delta_{0} \leq 1 / 3$ such that if a sequence $\mathbb{X}$ in $H$ is a $\delta$ lattice in the $\alpha$-parabolic sense with $0<\delta \leq \delta_{0}$, then $\mathbb{X}$ is a $\boldsymbol{b}_{\alpha}^{p}(\lambda)$-representing sequence of order $\kappa$.

Proof. Let $\mathbb{X}=\left\{\left(x_{j}, t_{j}\right)\right\}$ be a $\delta$-lattice in the $\alpha$-parabolic sense with $0<\delta \leq$ $1 / 3$. Here, constraints of $\delta$ will be imposed later. Since $\mathbb{X}$ is $\varepsilon$-separated in the $\alpha$-parabolic sense for some $\varepsilon>0$, Theorem 4.3 implies that $U_{p, \mathbb{X}}^{\kappa}: \ell^{p} \rightarrow \boldsymbol{b}_{\alpha}^{p}(\lambda)$ is bounded. Let $\left\{S_{j}\right\}$ be a pairwise disjoint covering of $H$ defined in (5.4). We define the operator $B_{p, \mathbb{X}}$ on $\boldsymbol{b}_{\alpha}^{p}(\lambda)$ by

$$
B_{p, \mathbb{X}} u:=\left\{t_{j}^{\left(\frac{n}{2 \alpha}+\lambda+1\right) \frac{1}{p}-\left(\frac{n}{2 \alpha}+1\right)} u\left(x_{j}, t_{j}\right) V\left(S_{j}\right)\right\} .
$$

Since $V\left(S_{j}\right) \leq C t_{j}^{\frac{n}{2 \alpha}+1}$ for all $j \geq 1$ and $\mathbb{X}$ is $\varepsilon$-separated in the $\alpha$-parabolic sense for some $\varepsilon>0$, Lemma 4.1 implies that $B_{p, \mathbb{X}}: \boldsymbol{b}_{\alpha}^{p}(\lambda) \rightarrow \ell^{p}$ is bounded. We also define the operator $A_{p, \mathbb{X}}^{\kappa}$ by

$$
\begin{aligned}
A_{p, \mathbb{X}}^{\kappa} u(x, t) & =C_{\kappa} U_{p, \mathbb{X}}^{\kappa} \circ B_{p, \mathbb{X}} \\
& =C_{\kappa} \sum_{j} t_{j}^{\kappa-1} u\left(x_{j}, t_{j}\right) \mathcal{D}_{t}^{\kappa} W^{(\alpha)}\left(x-x_{j}, t+t_{j}\right) V\left(S_{j}\right),
\end{aligned}
$$

where $C_{\kappa}$ is the constant defined in Theorem A. Then, $A_{p, \mathbb{X}}^{\kappa}: \boldsymbol{b}_{\alpha}^{p}(\lambda) \rightarrow \boldsymbol{b}_{\alpha}^{p}(\lambda)$ is bounded. We show that $\left\|I-A_{p, \mathbb{X}}^{\kappa}\right\|<1$ for all $\delta$ sufficiently small, where $I$ is the identity operator on $\boldsymbol{b}_{\alpha}^{p}(\lambda)$ and $\|\cdot\|$ is the operator norm. In fact, Lemma 2.5 implies that for $u \in \boldsymbol{b}_{\alpha}^{p}(\lambda)$ and $(x, t) \in H$,

$$
u(x, t)=C_{\kappa} \int_{H} u(y, s) \mathcal{D}_{t}^{\kappa} W^{(\alpha)}(x-y, t+s) s^{\kappa-1} d V(y, s) .
$$

Since $\left\{S_{j}\right\}$ is the pairwise disjoint covering of $H$, we obtain

$$
u(x, t)=C_{\kappa} \sum_{j} \int_{S_{j}} u(y, s) \mathcal{D}_{t}^{\kappa} W^{(\alpha)}(x-y, t+s) s^{\kappa-1} d V(y, s) .
$$

Hence by $(5.7)$ and $(5.5)$, we have $\left(I-A_{p, \mathbb{X}}^{\kappa}\right) u(x, t)=C_{\kappa}\left(\Pi_{1}(x, t)+\Pi_{2}(x, t)\right)$, where

$$
\begin{aligned}
\Pi_{1}(x, t)= & \sum_{j} \int_{S_{j}} u(y, s)\left(s^{\kappa-1} \mathcal{D}_{t}^{\kappa} W^{(\alpha)}(x-y, t+s)\right. \\
& \left.-t_{j}^{\kappa-1} \mathcal{D}_{t}^{\kappa} W^{(\alpha)}\left(x-x_{j}, t+t_{j}\right)\right) d V(y, s)
\end{aligned}
$$

and

$$
\Pi_{2}(x, t)=\sum_{j} \int_{S_{j}}\left(u(y, s)-u\left(x_{j}, t_{j}\right)\right) t_{j}^{\kappa-1} \mathcal{D}_{t}^{\kappa} W^{(\alpha)}\left(x-x_{j}, t+t_{j}\right) d V(y, s) .
$$


We estimate the function $\Pi_{1}$. Since $S_{j} \subset S_{\delta}^{(\alpha)}\left(x_{j}, t_{j}\right)$, Lemma 5.2 and the definition of $\left\{S_{j}\right\}$ imply that

$$
\begin{aligned}
\left|\Pi_{1}(x, t)\right| \leq & \sum_{j} \int_{S_{j}}|u(y, s)| \\
& \times\left|s^{\kappa-1} \mathcal{D}_{t}^{\kappa} W^{(\alpha)}(x-y, t+s)-t_{j}^{\kappa-1} \mathcal{D}_{t}^{\kappa} W^{(\alpha)}\left(x-x_{j}, t+t_{j}\right)\right| d V(y, s) \\
\leq & C\left(\delta+\delta^{\frac{1}{2 \alpha}}\right) \sum_{j} \int_{S_{j}} \frac{|u(y, s)| s^{\kappa-1}}{\left(t+s+|x-y|^{2 \alpha}\right)^{\frac{n}{2 \alpha}+\kappa}} d V(y, s) \\
= & C\left(\delta+\delta^{\frac{1}{2 \alpha}}\right) \int_{H} \frac{|u(y, s)| s^{\kappa-1}}{\left(t+s+|x-y|^{2 \alpha}\right)^{\frac{n}{2 \alpha}+\kappa}} d V(y, s) \\
= & C\left(\delta+\delta^{\frac{1}{2 \alpha}}\right) \Psi_{\alpha}^{\kappa, \kappa-1} u(x, t),
\end{aligned}
$$

where $\Psi_{\alpha}^{\kappa, \kappa-1}$ is the operator defined in Section 2. Therefore by Lemma 2.7, there exists a constant $C>0$ independent of $\delta$ such that

$$
\left\|\Pi_{1}\right\|_{L^{p}(\lambda)} \leq C\left(\delta+\delta^{\frac{1}{2 \alpha}}\right)\left\|\Psi_{\alpha}^{\kappa, \kappa-1} u\right\|_{L^{p}(\lambda)} \leq C\left(\delta+\delta^{\frac{1}{2 \alpha}}\right)\|u\|_{L^{p}(\lambda)}
$$

We estimate the function $\Pi_{2}$. Lemma 2.1 implies that

$$
\begin{aligned}
\left|\Pi_{2}(x, t)\right| & =\sum_{j} \int_{S_{j}}\left|u(y, s)-u\left(x_{j}, t_{j}\right)\right| t_{j}^{\kappa-1}\left|\mathcal{D}_{t}^{\kappa} W^{(\alpha)}\left(x-x_{j}, t+t_{j}\right)\right| d V(y, s) \\
& \leq C \sum_{j} \int_{S_{j}} \frac{\left|u(y, s)-u\left(x_{j}, t_{j}\right)\right| t_{j}^{\kappa-1}}{\left(t+t_{j}+\left|x-x_{j}\right|^{2 \alpha}\right)^{\frac{n}{2 \alpha}+\kappa}} d V(y, s) .
\end{aligned}
$$

Since $S_{j} \subset S_{\delta}^{(\alpha)}\left(x_{j}, t_{j}\right) \subseteq S_{1 / 3}^{(\alpha)}\left(x_{j}, t_{j}\right)$, there exists a constant $C>0$ independent of $\delta$ such that

$$
C^{-1} t_{j}<s<C t_{j} \text { and } t+s+|x-y|^{2 \alpha} \leq C\left(t+t_{j}+\left|x-x_{j}\right|^{2 \alpha}\right)
$$

for all $(y, s) \in S_{j}$. Furthermore by (5.6) and Lemma 5.2, there exists a constant $C>0$ independent of $\delta$ such that

$$
\begin{aligned}
& \left|u(y, s)-u\left(x_{j}, t_{j}\right)\right| \\
\leq & C \int_{H}|u(z, r)| \\
& \times\left|\mathcal{D}_{t}^{\lambda+2} W^{(\alpha)}(y-z, s+r)-\mathcal{D}_{t}^{\lambda+2} W^{(\alpha)}\left(x_{j}-z, t_{j}+r\right)\right| r^{\lambda+1} d V(z, r) \\
\leq & C\left(\delta+\delta^{\frac{1}{2 \alpha}}\right) \int_{H} \frac{|u(z, r)| r^{\lambda+1}}{\left(s+r+|y-z|^{2 \alpha}\right)^{\frac{n}{2 \alpha}+\lambda+2}} d V(z, r)
\end{aligned}
$$


for all $(y, s) \in S_{j}$. Hence, (5.8) and (5.9) imply that

$$
\begin{aligned}
\left|\Pi_{2}(x, t)\right| \leq & C\left(\delta+\delta^{\frac{1}{2 \alpha}}\right) \sum_{j} \int_{S_{j}} \int_{H} \frac{|u(z, r)| r^{\lambda+1}}{\left(s+r+|y-x|^{2 \alpha}\right)^{\frac{n}{2 \alpha}+\lambda+2}} d V(z, r) \\
& \times \frac{s^{\kappa-1}}{\left(t+s+|x-y|^{2 \alpha}\right)^{\frac{n}{2 \alpha}+\kappa}} d V(y, s) \\
\leq & C\left(\delta+\delta^{\frac{1}{2 \alpha}}\right) \int_{H} \int_{H} \frac{|u(z, r)| r^{\lambda+1}}{\left(s+r+|y-x|^{2 \alpha}\right)^{\frac{n}{2 \alpha}+\lambda+2}} d V(z, r) \\
& \times \frac{s^{\kappa-1}}{\left(t+s+|x-y|^{2 \alpha}\right)^{\frac{n}{2 \alpha}+\kappa}} d V(y, s) \\
\leq & C\left(\delta+\delta^{\frac{1}{2 \alpha}}\right) \Psi_{\alpha}^{\kappa, \kappa-1}\left(\Psi_{\alpha}^{\lambda+2, \lambda+1} u\right)(x, t) .
\end{aligned}
$$

Therefore by Lemma 2.7 , we obtain

$$
\begin{aligned}
\left\|\Pi_{2}\right\|_{L^{p}(\lambda)} & \leq C\left(\delta+\delta^{\frac{1}{2 \alpha}}\right)\left\|\Psi_{\alpha}^{\kappa, \kappa-1}\left(\Psi_{\alpha}^{\lambda+2, \lambda+1} u\right)\right\|_{L^{p}(\lambda)} \\
& \leq C\left(\delta+\delta^{\frac{1}{2 \alpha}}\right)\left\|\Psi_{\alpha}^{\lambda+2, \lambda+1} u\right\|_{L^{p}(\lambda)} \leq C\left(\delta+\delta^{\frac{1}{2 \alpha}}\right)\|u\|_{L^{p}(\lambda)} .
\end{aligned}
$$

Hence, there exists $0<\delta_{0} \leq 1 / 3$ such that $\left\|I-A_{p, \mathbb{X}}^{\kappa}\right\|<1$ for all $0<\delta \leq \delta_{0}$. This completes the proof.

\section{6. $b_{\alpha}^{p}(\lambda)$-sampling sequences with fractional derivatives}

In this section, we introduce $\boldsymbol{b}_{\alpha}^{p}(\lambda)$-sampling sequences, and we show that $\boldsymbol{b}_{\alpha}^{p}(\lambda)$-representing sequences are closely related to $\boldsymbol{b}_{\alpha}^{p}(\lambda)$-sampling sequences. We give the definition of $\boldsymbol{b}_{\alpha}^{p}(\lambda)$-sampling sequences. Let $0<\alpha \leq 1,1 \leq p<\infty$, $\lambda>-1, \nu \in \mathbb{R}$, and $\mathbb{X}=\left\{\left(x_{j}, t_{j}\right)\right\}$ be a sequence in $H$. We say that $\mathbb{X}$ is a $\boldsymbol{b}_{\alpha}^{p}(\lambda)$-sampling sequence of order $\nu$ if there exists a constant $C>0$ such that

$$
C^{-1}\|u\|_{L^{p}(\lambda)}^{p} \leq \sum_{j} t_{j}^{\frac{n}{2 \alpha}+\lambda+1+\nu p}\left|\mathcal{D}_{t}^{\nu} u\left(x_{j}, t_{j}\right)\right|^{p} \leq C\|u\|_{L^{p}(\lambda)}^{p}
$$

for all $u \in \boldsymbol{b}_{\alpha}^{p}(\lambda)$. In other words, $\mathbb{X}$ is a $\boldsymbol{b}_{\alpha}^{p}(\lambda)$-sampling sequence if the operator $T_{p, \mathbb{X}}^{\nu}: \boldsymbol{b}_{\alpha}^{p}(\lambda) \rightarrow \ell^{p}$ is bounded and bounded below, where $T_{p, \mathbb{X}}^{\nu}=T_{p, \mathbb{X}}^{0, \nu}$ is defined in Section 4. In order to show the main theorem of this section, we present the following lemma, which is already given in the proof of Theorem 4.3.

Lemma 6.1. Let $0<\alpha \leq 1,1<p<\infty, \lambda>-1$, and $\kappa>\frac{\lambda+1}{p}$. Furthermore, let $\mathbb{X}$ be a sequence in $H$, and $q$ the exponent conjugate to $p$. If $U_{p, \mathbb{X}}^{\kappa}: \ell^{p} \rightarrow$ $\boldsymbol{b}_{\alpha}^{p}(\lambda)$ is bounded, then

$$
\left(U_{p, \mathbb{X}}^{\kappa}\right)^{*} u=\frac{1}{C_{\lambda+1}} T_{q, \mathbb{X}}^{\kappa-(\lambda+1)} u
$$

for all $u \in \boldsymbol{b}_{\alpha}^{q}(\lambda)$.

We also need the following lemma, which is given in [10]. 
Lemma 6.2 (Theorem 4.13 of [10]). Let $X$ and $Y$ be Banach spaces. Furthermore, let $T$ be a bounded operator from $X$ into $Y$. Then, the following statements are equivalent:

(1) $T(X)=Y$.

(2) There exists a constant $C>0$ such that $\left\|T^{*} y^{*}\right\| \geq C\left\|y^{*}\right\|$ for all $y^{*} \in Y^{*}$.

Now, we show the main theorem of this section.

Theorem 6.3. Let $0<\alpha \leq 1,1<p<\infty, \lambda>-1$, and $\kappa>\frac{\lambda+1}{p}$. Furthermore, let $\mathbb{X}$ be a sequence in $H$, and $q$ the exponent conjugate to $p$. Then, the following conditions are equivalent:

(1) $\mathbb{X}$ is a $\boldsymbol{b}_{\alpha}^{p}(\lambda)$-representing sequence of order $\kappa$.

(2) $\mathbb{X}$ is a $\boldsymbol{b}_{\alpha}^{q}(\lambda)$-sampling sequence of order $\kappa-(\lambda+1)$.

Proof. $(1) \Rightarrow(2)$. We assume that $\mathbb{X}$ is a $\boldsymbol{b}_{\alpha}^{p}(\lambda)$-representing sequence of order $\kappa$, that is, $U_{p, \mathbb{X}}^{\kappa}: \ell^{p} \rightarrow \boldsymbol{b}_{\alpha}^{p}(\lambda)$ is bounded and $U_{p, \mathbb{X}}^{\kappa}\left(\ell^{p}\right)=\boldsymbol{b}_{\alpha}^{p}(\lambda)$. Then, Lemmas $2.6,6.1$, and 6.2 imply that

$$
\left\|T_{q, \mathbb{X}}^{\kappa-(\lambda+1)} u\right\|_{\ell^{q}}=C\left\|\left(U_{p, \mathbb{X}}^{\kappa}\right)^{*} u\right\|_{\ell^{q}} \geq C\|u\|_{L^{q}(\lambda)}
$$

for all $u \in \boldsymbol{b}_{\alpha}^{q}(\lambda)$. Therefore, $T_{q, \mathbb{X}}^{\kappa-(\lambda+1)}: \boldsymbol{b}_{\alpha}^{q}(\lambda) \rightarrow \ell^{q}$ is bounded below. Moreover, by Lemma 4.1 and Theorem $4.3, T_{q, \mathbb{X}}^{\nu}: \boldsymbol{b}_{\alpha}^{q}(\lambda) \rightarrow \ell^{q}$ is bounded for each $\nu>-\frac{\lambda+1}{q}$. Hence, $\mathbb{X}$ is a $\boldsymbol{b}_{\alpha}^{q}(\lambda)$-sampling sequence of order $\kappa-(\lambda+1)$.

$(2) \Rightarrow(1)$. We assume that $\mathbb{X}$ is a $\boldsymbol{b}_{\alpha}^{q}(\lambda)$-sampling sequence of order $\kappa-$ $(\lambda+1)$, that is, $T_{q, \mathbb{X}}^{\kappa-(\lambda+1)}: \boldsymbol{b}_{\alpha}^{q}(\lambda) \rightarrow \ell^{q}$ is bounded and bounded below. Then, by Lemma 4.1 and Theorem 4.3, $U_{p, \mathbb{X}}^{\kappa}: \ell^{p} \rightarrow \boldsymbol{b}_{\alpha}^{p}(\lambda)$ is bounded. Therefore, Lemma 6.1 implies that

$$
\left\|\left(U_{p, \mathbb{X}}^{\kappa}\right)^{*} u\right\|_{\ell^{q}}=C\left\|T_{q, \mathbb{X}}^{\kappa-(\lambda+1)} u\right\|_{\ell^{q}} \geq C\|u\|_{L^{q}(\lambda)}
$$

for all $u \in \boldsymbol{b}_{\alpha}^{q}(\lambda)$. Hence, by Lemmas 2.6 and 6.2 , we obtain $U_{p, \mathbb{X}}^{\kappa}\left(\ell^{p}\right)=\boldsymbol{b}_{\alpha}^{p}(\lambda)$. Thus, $\mathbb{X}$ is a $\boldsymbol{b}_{\alpha}^{p}(\lambda)$-representing sequence of order $\kappa$.

\section{References}

[1] B. R. Choe and H. Yi, Representations and interpolations of harmonic Bergman functions on half-spaces, Nagoya Math. J. 151 (1998), 51-89.

[2] R. R. Coifman and R. Rochberg, Representation theorems for holomorphic and harmonic functions in $L^{p}$, Representation theorems for Hardy spaces, pp. 11-66, Astérisque, 77, Soc. Math. France, Paris, 1980.

[3] Y. Hishikawa, Fractional calculus on parabolic Bergman spaces, Hiroshima Math. J. 38 (2008), no. 3, 471-488.

[4] Y. Hishikawa, M. Nishio, and M. Yamada, A conjugate system and tangential derivative norms on parabolic Bergman spaces, Hokkaido Math. J. 39 (2010), no. 1, 85-114.

[5] M. Nishio, K. Shimomura, and N. Suzuki, $\alpha$-parabolic Bergman spaces, Osaka J. Math. 42 (2005), no. 1, 133-162.

[6] M. Nishio, N. Suzuki, and M. Yamada, Carleson inequalities on parabolic Bergman spaces, to appear in Tohoku Math. J.

[7] _ Interpolating sequences of parabolic Bergman spaces, Potential Anal. 28 (2008), no. $4,357-378$. 
[8] K. Nam, Representations and interpolations of weighted harmonic Bergman functions, Rocky Mountain J. Math. 36 (2006), no. 1, 237-263.

[9] W. Ramey and H. Yi, Harmonic Bergman functions on half-spaces, Trans. Amer. Math. Soc. 348 (1996), no. 2, 633-660.

[10] W. Rudin, Functional Analysis, Second edition, International Series in Pure and Applied Mathematics. McGraw-Hill, Inc., New York, 1991.

DEPARTMENT OF GENERAL EDUCATION

Gifu National College of Technology

KAMIMAKUWA 2236-2, Gifu 501-0495, JAPAN

E-mail address: yosuke-h@gifu-nct.ac.jp 